\title{
Cam-Clay plasticity, Part VIII: A constitutive framework for porous materials with evolving internal structure
}

\author{
Ronaldo I. Borja* · Jinhyun Choo \\ Department of Civil and Environmental Engineering, Stanford University, \\ Stanford, CA 94305, USA. \\ ${ }^{*}$ Corresponding author, E-mail: borja@stanford.edu
}

\begin{abstract}
Summary. Natural geomaterials often exhibit pore size distributions with two dominant porosity scales. Examples include fractured rocks where the dominant porosities are those of the fractures and rock matrix, and aggregated soils where the dominant porosities are those of the micropores and macropores. We develop a constitutive framework for this type of materials that covers both steady-state and transient fluid flow responses. The framework relies on a thermodynamically consistent effective stress previously developed for porous media with two dominant porosity scales. We show that this effective stress is equivalent to the weighted sum of the individual effective stresses in the micropores and macropores, with the weighting done according to the pore fractions. This partitioning of the effective stress into two single-porosity effective stresses allows fluid pressure dissipation at the macropores and micropores to be considered separately, with important implications for individual characterization of the hardening responses at the two pore scales. Experimental data suggest that the constitutive framework captures the laboratory responses of aggregated soils more accurately than other models previously reported in the literature. Numerical simulations of boundary-value problems reveal the capability of the framework to capture the effect of secondary compression as the micropores discharge fluids into the macropores.
\end{abstract}

Keywords: coupled problem $\cdot$ double porosity $\cdot$ effective stress $\cdot$ mixture theory

\section{Introduction}

Many natural geomaterials such as aggregated soils and fissured rocks exhibit pore size distributions with two dominant values of porosity $[1,2,26,28,31$, 55]. In fissured rocks the two scales of porosity are those of the rock matrix and fractures $[11,61,64,68,74,77,79,83]$, whereas in aggregated soils,

(C) 2016. This manuscript version is made available under the Elsevier user license http://www.elsevier.com/open-access/userlicense/1.0/ 
the two scales of porosity pertain to those of the micropores (intra-aggregate pores) and macropores (inter-aggregate pores), which can be filled up with one or more types of fluid $[9,27,29,60,65-67,82]$. Of interest in these socalled 'double-porosity' media is the evolution of the internal structure and the proportional changes in the micro- and macro-porosities during the course of loading [44, 48, 49,53,54]. Using a combination of tomography techniques and image analysis on aggregated soils, Koliji et al. [50] showed that the volume and structure of the macropores are strongly affected by the mechanical loading, while the corresponding changes in the micropores are almost insignificant. They showed that significant changes in the structure occurs with plastic straining, leading to an evolution law that links the macroporosity alteration to plastic strain [51, 52].

Early applications of double porosity concepts have focused on the behavior of fissured rocks with respect to their ability to transmit fluid through the matrix pores and fractures $[6,7]$. Subsequent developments have addressed fluid flow through aggregated soils and their implications for the so-called 'effective stress' in materials with double porosity [20, 45, 46, 59]. Many of the proposed expressions for the effective stress in two-porosity fields lacked a thermodynamic basis that has been the cornerstone of similar developments for single-porosity problems [14, 15, 35, 36, 40-43]. An exception is the expression for the effective stress in two-porosity fields developed by Borja and Koliji [13], which emanated directly from mixture theory and continuum principles of thermodynamics. Despite these efforts to develop a suitable measure of effective stress, however, much work remains to be done with respect to mathematically delineating the evolution of internal structure and how it impacts fluid flow through the micropores and macropores of materials with two dominant porosity scales.

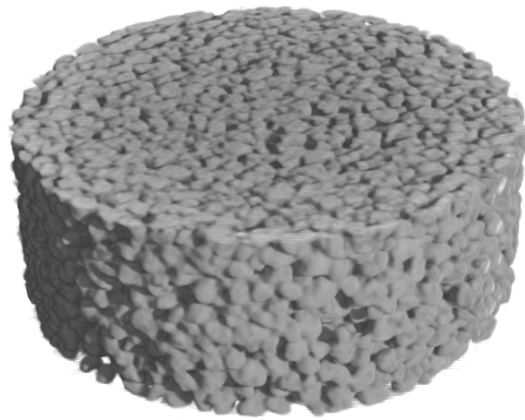

Fig. 1. Reconstructed computed tomography volume of an aggregated silty clay, diameter $=80 \mathrm{~mm}$, height $=35 \mathrm{~mm}$. The aggregates are composed of much smaller silty clay solid particles with intra-aggregate pores. Visible spaces between aggregates are the inter-aggregate pores. After Koliji [51] and Borja and Koliji [13]. 
This work focuses more specifically on the behavior of aggregated soils depicted by the computed tomography volume of Fig. 1 . The volume contains aggregates of reconstituted soil with much smaller intra-aggregate pores (micropores), as compared to the much larger inter-aggregate pores (macropores) that are very visible in the figure. For the sake of clarity, we use the concept of 'dual permeability' in which fluid may flow not only through the larger macropores but also through the smaller micropores, as well as between contacting aggregates through their contact areas [32,69]. Fluid may also be exchanged between the micropores and macropores through the surface of the aggregates exposed to the inter-aggregate pores [22]. The macropores define the internal structure of the volume. As the volume is compressed both the macropores and micropores can compact, but the compaction of the macropores is more dominant during the early stages of compression. As the macropores are squeezed the internal structure disappears [18], leaving behind a volume with only the micropores and having a similar material behavior to that of the reconstituted soil.

A challenge with modeling double porosity media is that there are two compressibility responses that must be captured within the same constitutive framework - that of the reconstituted material with an evolving internal structure, and that of the reconstituted material itself $[4,48,49]$. Because the internal structure disappears with collapse of the macropores, the response of the system with internal structure must necessarily approach the response of the reconstituted material itself at high compressive loads. This requires a nonlinear continuum mechanics approach accommodating an evolving configuration in general and an evolving macro-porosity in particular. If the macropores and micropores are filled with fluids that are free to migrate within and outside their pore scales, then the constitutive framework must also accommodate two volume constraints imposed by the two pore scales. Furthermore, in the context of critical state theory $[3,18]$ the preconsolidation stresses at the two pore scales must also be tracked concurrently with fluid pressure dissipation.

This paper takes a nonlinear continuum mechanics approach to accommodate an evolving internal structure by partitioning the overall effective stress of double-porosity media into two single-porosity effective stresses appropriate for the macropores and micropores. In doing so, fluid pressure dissipation at the two pore scales as well as the accompanying hardening of the preconsolidation stresses can be tracked individually. The core aspect of the formulation is the expression for the rate of change of internal energy for double-porosity media [13], which determines the energy-conjugate pairing of the relevant constitutive variables. The constitutive model is cast within the mixed finite element framework. Without loss of generality, we assume throughout this paper that the two pore scales are fully saturated with the same type of fluid (e.g. water), and leave the subject of partial saturation, in which the two pore scales may be filled with more than one type of fluid, for future work. 


\section{General formulation}

This section focuses on the kinematics of deformation of a porous solid with double porosity. The general notion of compressibility in the context of double porosity is also introduced, along with a formulation of elastoplasticity in terms of the effective Kirchhoff stress that incorporates an evolving internal structure, herein represented by the pore fraction $\psi$.

\subsection{Solid kinematics}

Consider an aggregated soil with elementary volume shown schematically in Fig. 2. The motion of the solid is defined by the deformation $\boldsymbol{x}=\boldsymbol{\phi}(\boldsymbol{X}, t)$, with deformation gradient $\boldsymbol{F}=\partial \phi / \partial \boldsymbol{X}$. The solid deformation carries with it both the macropore and micropore volumes quantified by the void ratios

$$
e_{m}(\boldsymbol{X}, t)=\frac{\mathrm{d} V_{v m}}{\mathrm{~d} V_{s}}, \quad e_{M}(\boldsymbol{X}, t)=\frac{\mathrm{d} V_{v M}}{\mathrm{~d} V_{s}},
$$

where $\mathrm{d} V_{v m}$ and $\mathrm{d} V_{v M}$ are the volumes of the micropores and macropores, respectively, and $\mathrm{d} V_{s}$ is the volume of solid contained in the elementary volume. We also define the following volume fractions

$$
\phi^{s}(\boldsymbol{X}, t)=\frac{\mathrm{d} V_{s}}{\mathrm{~d} V}, \quad \psi(\boldsymbol{X}, t)=\frac{\mathrm{d} V_{v m}}{\mathrm{~d} V_{v}},
$$

where $\mathrm{d} V_{v}=\mathrm{d} V_{v m}+\mathrm{d} V_{v M}$ is the total volume of the void within the total volume $\mathrm{d} V$ of the mixture. In this last equation, $\phi^{s}$ is the solid volume fraction while $\psi$ is the fraction of the void occupied by the micropores.

The specific volume of the micropores is given by

$$
v_{m}(\boldsymbol{X}, t)=1+e_{m}(\boldsymbol{X}, t),
$$

which is the total volume of the aggregate per unit solid volume. The overall specific volume is

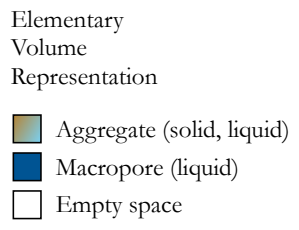

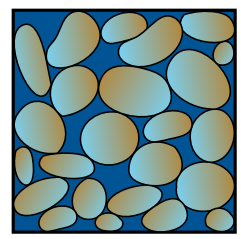

(a)

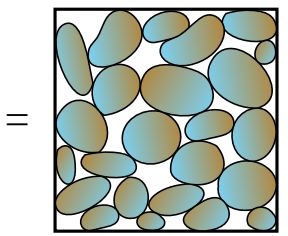

(b)

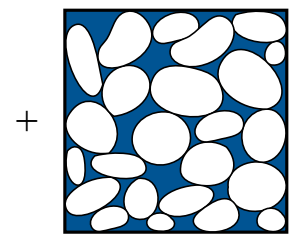

(c)

Fig. 2. Schematic representation of aggregated soil with double porosity: (a) total saturated mixture of solid and liquid; (b) aggregate of solid and liquid; and (c) macropores completely filled with liquid. After Koliji [51] and Borja and Koliji [13]. 


$$
v(\boldsymbol{X}, t)=v_{m}(\boldsymbol{X}, t)+e_{M}(\boldsymbol{X}, t),
$$

which is the total volume of the mixture per unit solid volume. In aggregated soils, the aggregate is typically associated with a reconstituted material with no internal structure such as clay. On the other hand, the internal structure of the soil is defined by the macropores. We note that $v(\boldsymbol{X}, t) \rightarrow v_{m}(\boldsymbol{X}, t)$ as $e_{M}(\boldsymbol{X}, t) \rightarrow 0$, which means that the internal structure of the soil disappears when the macropores collapse.

If the solid does not exchange mass with the fluid, then its motion should be mass conserving. Dropping the spatial and temporal arguments $\boldsymbol{X}$ and $t$ for brevity, this means that

$$
\overline{J \phi^{s} \rho_{s}}=0,
$$

where $J=\operatorname{det}(\boldsymbol{F})$ is the Jacobian of the solid motion, $\rho_{s}$ is the intrinsic mass density of the solid, and the superimposed dot denotes a material time derivative with respect to the solid motion. Without loss of generality we shall assume that the solid constitutent is incompressible and take $\rho_{s}=$ constant, which yields the following evolution of the solid volume fraction

$$
\phi^{s}=\frac{\phi_{0}^{s}}{J},
$$

where $\phi_{0}^{s}$ is the volume fraction of the solid when $J=J_{0}=1$. Thus, the evolution of $\phi^{s}$ can be determined from the variation of the Jacobian $J$ alone.

The evolutions of the specific volumes are given by

$$
v=\frac{1}{\phi^{s}}=\frac{J}{\phi_{0}^{s}}
$$

for the macropores, and

$$
v_{m}=1+\psi \frac{1-\phi^{s}}{\phi^{s}}=1-\psi+\frac{\psi J}{\phi_{0}^{s}}
$$

for the micropores. Note that the expression for $v$ is determined by $J$ alone, whereas the expression for $v_{m}$ also contains the pore fraction $\psi$ that must be determined separately. The difference between the two specific volumes is the void ratio of the macropores,

$$
e_{M}=v-v_{m}=(1-\psi) \frac{1-\phi^{s}}{\phi^{s}}=(1-\psi)\left(\frac{J}{\phi_{0}^{s}}-1\right) .
$$

\subsection{Effective stress and the first law}

For double-porosity media whose macropores (denoted by index $M$ ) and micropores (denoted by index $m$ ) are saturated with incompressible fluid (e.g. water), the rate of change of internal energy per unit volume takes the form $[13,24]$ 


$$
J \rho \dot{e}=\langle\overline{\boldsymbol{\tau}}, \boldsymbol{d}\rangle+\sum_{i=M, m}\left\langle\tilde{\boldsymbol{v}}_{i}, \phi^{i}, p_{i}\right\rangle-\sum_{i=M, m}\left\langle c^{i}, p_{i}, \tilde{\boldsymbol{v}}_{i}\right\rangle-\left\langle\left(1-\phi^{s}\right), \pi, \dot{\psi}\right\rangle,
$$

where the symbol $\langle\circ, \ldots, \circ\rangle$ denotes energy-conjugate pairing in product form. The notations are as follows: $\dot{e}$ is the rate of change of internal energy per unit total mass of the mixture, $\rho$ is the total mass density of the mixture, $\bar{\tau}$ is the effective Kirchhoff stress tensor, $\boldsymbol{d}$ is the rate of deformation tensor, $\tilde{\boldsymbol{v}}_{i}$ is the relative velocity of fluid in pore scale $i$ with respect to solid, $\phi^{i}$ is the volume fraction of pore scale $i, p_{i}$ is the intrinsic Kirchhoff fluid pressure in pore scale $i, c^{i}$ is a mass exchange term satisfying the closure condition $c^{M}+c^{m}=0, \phi^{s}$ is the volume fraction of solid (defined previously), $\psi$ is the void fraction for the micropores (also defined previously), and

$$
\pi=p_{M}-p_{m}
$$

is the Kirchhoff fluid pressure jump between the two pore scales. The Kirchhoff stresses and pressures differ from the Cauchy definitions by the factor $J$, i.e., $\overline{\boldsymbol{\tau}}=J \overline{\boldsymbol{\sigma}}$ and $p_{i}=J \theta_{i}$, where $\overline{\boldsymbol{\sigma}}$ is the Cauchy effective stress tensor and $\theta_{i}$ is the Cauchy fluid pressure in pore scale $i$. The foregoing expression for the rate of change of internal energy yields four groups of constitutive variables that imply four types of constitutive relations. We shall elaborate these pairs of constitutive relations in a later section, but for now we focus on the effective stress $\overline{\boldsymbol{\sigma}}$ and the fourth term in the energy equation involving the pressure jump $\pi$.

The effective Kirchhoff stress tensor is given by the expression [13]

$$
\overline{\boldsymbol{\tau}}=\boldsymbol{\tau}+\bar{p} \mathbf{1}
$$

where $\boldsymbol{\tau}$ is the total Kirchhoff stress tensor, $\mathbf{1}$ is the second-order identity tensor, and $\bar{p}$ is the Kirchhoff mean fluid pressure given by

$$
\bar{p}=\psi p_{m}+(1-\psi) p_{M}
$$

Here, we take the Biot coefficient equal to unity following the earlier assumption of an incompressible solid constituent. Rewriting the total Kirchhoff stress tensor as $\boldsymbol{\tau}=\psi \boldsymbol{\tau}+(1-\psi) \boldsymbol{\tau}$, we find that

$$
\overline{\boldsymbol{\tau}}=\psi \overline{\boldsymbol{\tau}}_{m}+(1-\psi) \overline{\boldsymbol{\tau}}_{M},
$$

where

$$
\overline{\boldsymbol{\tau}}_{m}=\boldsymbol{\tau}+p_{m} \mathbf{1}, \quad \overline{\boldsymbol{\tau}}_{M}=\boldsymbol{\tau}+p_{M} \mathbf{1}
$$

are the single-porosity effective Kirchhoff stress tensors at each pore scale [14]. Note that these two stress tensors are not independent, but instead are related by the equation

$$
\overline{\boldsymbol{\tau}}_{m}=\overline{\boldsymbol{\tau}}_{M}-\pi \mathbf{1} .
$$

In other words, the two single-porosity effective stresses are simply translated on the hydrostatic axis by the fluid pressure jump $\pi$. 
We now consider an isotropic state of stress and denote the mean normal effective stresses by the symbols $\bar{\tau}_{m}=\operatorname{tr}\left(\overline{\boldsymbol{\tau}}_{m}\right) / 3$ and $\bar{\tau}_{M}=\operatorname{tr}\left(\overline{\boldsymbol{\tau}}_{M}\right) / 3$. Equation (16) thus reduces to

$$
\pi=\bar{\tau}_{M}-\bar{\tau}_{m} .
$$

When the macropores and micropores are both yielding, the effective mean normal stresses may be replaced by their respective preconsolidation stresses, i.e., $\bar{\tau}_{m}=p_{c m}$ and $\bar{\tau}_{M}=p_{c M}$. The overall preconsolidation stress for the aggregated soil is then equal to

$$
\bar{\tau}=\frac{1}{3} \operatorname{tr}(\overline{\boldsymbol{\tau}})=\psi \bar{\tau}_{m}+(1-\psi) \tau_{M}:=\bar{p}_{c},
$$

where

$$
\bar{p}_{c}=\psi p_{c m}+(1-\psi) p_{c M} .
$$

When the material is yielding the fluid pressure jump is also equal to the jump in the preconsolidation pressures, i.e.,

$$
\pi=p_{c M}-p_{c m} .
$$

The fourth energy-conjugate term on the right-hand side of equation (10) suggests compressibility laws of the form $p_{c m}=p_{c m}\left(\phi^{s}, \psi\right)$ and $p_{c M}=$ $p_{c M}\left(\phi^{s}, \psi\right)$, which give

$$
\pi=p_{c M}\left(\phi^{s}, \psi\right)-p_{c m}\left(\phi^{s}, \psi\right) \equiv g\left(\phi^{s}, \psi\right) .
$$

For incompressible solid constituents, variations in void fraction $\phi^{s}$ are associated with changes in the volume of the pores. If such changes are irreversible, or if the reversible component is insignificant, then compressibility laws of the following form may be used

$$
\pi=\tilde{g}\left(J^{\mathrm{p}}, \psi\right),
$$

where $J^{\mathrm{p}}$ is the plastic component of $J$. The next section elaborates how these compressibility laws impact the evolution of the internal structure of a material with two porosity scales, as well as how they compare with other compressibility laws developed for aggregated soils such as Abist silt [52] and Corinth marl [4].

\subsection{Compressibility laws}

Consider a 'normally consolidated' porous material where both the macropores and micropores are yielding such that the internal structure evolves according to the following compressibility laws,

$$
v_{m}=f\left(p_{c m}\right), \quad e_{M}=g\left(p_{c M}\right) .
$$


The inverse relations are given by

$$
p_{c m}=f^{-1}\left(v_{m}\right), \quad p_{c M}=g^{-1}\left(e_{M}\right) .
$$

Note that $p_{c m}$ and $p_{c M}$ vary with $\phi^{s}$ and $\psi$ through $v_{m}$ and $e_{M}$, respectively, see equations (8) and (9).

Now, assume that there is no pore pressure jump between the micropores and macropores so that $\pi=0$. This condition holds if both pore scales are dry, or if the load is applied at a sufficiently slow rate that no pore pressure difference develops between the two pore scales. Setting $p_{c m}=p_{c M} \equiv \bar{p}_{c}$ determines the evolution of the micropore fraction $\psi$ for a given evolution of $\phi^{s}$ (or $J$ ). Note that the compressibility laws are generally of nonlinear form, so a closed-form expression for $\psi$ may not be readily available. However, in principle one can always calculate $\psi$ numerically (by iteration, for example). Having determined the variation of $\psi$ with $J$, one can then substitute it back into the expression for the preconsolidation stress to obtain the corresponding variation of $\bar{p}_{c}$ as a function of $J$. Thus, both $\psi$ and $\bar{p}_{c}$ can be determined from the given time history of $J$.

Now, suppose that instead of $J$ we are given the time history of the effective isotropic stress $\bar{\tau} \equiv \bar{p}_{c}$, and assume once again that $\pi=0$. The goal is to find $\psi$ and $J$ such that

$$
p_{c m}=f^{-1}\left(v_{m}(\psi, J)\right) \equiv \bar{p}_{c},
$$

and

$$
p_{c M}=g^{-1}\left(e_{M}(\psi, J)\right) \equiv \bar{p}_{c} .
$$

We have two equations in two unknowns, so we can determine the variations of $J$ and $\psi$ from the given time history of $\bar{p}_{c}$. If $\pi \neq 0$, then its time history must be prescribed to determine the evolution of the micropore fraction $\psi$.

The specific compressibility laws of interest are of the following form. For the micropores, we have

$$
\frac{\dot{v}_{m}}{v_{m}}=-c_{c} \frac{\dot{p}_{c m}}{p_{c m}}, \quad \dot{\varepsilon}_{v}^{e}=-c_{r} \frac{\dot{p}_{c m}}{p_{c m}},
$$

where $c_{c}$ and $c_{r}$ are total and elastic compressibility indices, respectively, and $\varepsilon_{v}^{e}$ is the elastic logarithmic volumetric strain. Direct time integration gives

$$
p_{c m}=\left(p_{c m}\right)_{n} \exp \left[-\frac{\ln \left(v_{m} / v_{m n}\right)-\left(\varepsilon_{v}^{e}-\varepsilon_{v n}^{e}\right)}{c_{c}-c_{r}}\right],
$$

where subscript $n$ pertains to reference value at time $t_{n}$, and $v_{m}=v_{m}(\psi, v)$ from equation (8). This equation is equivalent to the bilogarithmic compressibility law proposed by Butterfield [19] for reconstituted soils.

For the macropores, we consider a similar bilogarithmic compressibility law of the form

$$
\frac{\dot{e}_{M}}{e_{M}}=-c_{M} \frac{\dot{p}_{c M}}{p_{c M}}
$$


where $c_{M}$ is the compressibility index determined from the macropore compressibility curve, which is the difference between the total and micropore compressibility curves. Integrating yields

$$
p_{c M}=\left(p_{c M}\right)_{n} \exp \left[-\frac{\ln \left(e_{M} / e_{M n}\right)}{c_{M}}\right] .
$$

This law predicts an asymptotic decay in the sense $v \rightarrow v_{m}$ as $-p_{c M} \rightarrow \infty$. Later we shall validate these compressibility laws against experimental data on aggregated soils.

\subsection{Continuum formulation}

This section formulates the rate constitutive equations for double-porosity continua with an evolving internal structure represented by the scalar variable $\psi$. The formulation is done in abstract form to elucidate the essential ingredients of the theory. More specific elements of the theory are presented in the next section. Our point of departure is the rate constitutive equation in effective Kirchhoff stress space of the form (see equation (5.82) of [12])

$$
\dot{\overline{\boldsymbol{\tau}}}=\boldsymbol{\alpha}^{\mathrm{e}}:\left(\boldsymbol{d}-\dot{\lambda} \frac{\partial G}{\partial \overline{\boldsymbol{\tau}}}\right),
$$

where $\boldsymbol{d}$ is the symmetric part of the velocity gradient tensor $\boldsymbol{l}$, called the rateof-deformation tensor, $G$ is the plastic potential function, $\dot{\lambda} \geq 0$ is the plastic consistency parameter, and $\boldsymbol{\alpha}^{\mathrm{e}}$ is a rank-four elastic tangent tensor analogous to the elastic constitutive tensor $\boldsymbol{c}^{\mathrm{e}}$ in infinitesimal theory. We should note that $\boldsymbol{\alpha}^{\mathrm{e}}$ is amenable to spectral decomposition (see equation (5.88) of [12]), and contains the initial stress terms necessary to make the transformation objective with respect to rigid-body rotation. Because $\overline{\boldsymbol{\tau}}$ is a symmetric tensor, the plastic flow direction $\partial G / \partial \overline{\boldsymbol{\tau}}$ is also a symmetric tensor, which means that the plastic spin is ignored.

Next, we consider a yield function of the form $F\left(\overline{\boldsymbol{\tau}}, \bar{p}_{c}\right) \leq 0$. Assuming the yield condition $F=0$ is satisfied, the consistency condition for continued yielding takes the form

$$
\dot{F}=0 \Rightarrow \frac{\partial F}{\partial \bar{\tau}}: \dot{\bar{\tau}}+\frac{\partial F}{\partial \bar{p}_{c}} \dot{\bar{p}}_{c}=0
$$

where

$$
\dot{\bar{p}}_{c}=\frac{\partial \bar{p}_{c}}{\partial \psi} \dot{\psi}+\frac{\partial \bar{p}_{c}}{\partial J^{\mathrm{p}}} \dot{J}^{\mathrm{p}} \equiv \alpha_{1} \dot{\psi}+\alpha_{2} \dot{\lambda} .
$$

In this last equation, we take $\dot{J}^{\mathrm{p}} \propto \dot{\lambda}$ and $\alpha_{2}$ is some scalar coefficient of the consistency parameter $\dot{\lambda}$.

We now eliminate $\dot{\psi}$ by appealing to equation (22). Taking the rates gives

$$
\dot{\pi}=\frac{\partial \tilde{g}}{\partial \psi} \dot{\psi}+\frac{\partial \tilde{g}}{\partial J^{\mathrm{p}}} \dot{J}^{\mathrm{p}} \equiv \beta_{1} \dot{\psi}+\beta_{2} \dot{\lambda}
$$


Here, we note that $\dot{\pi}$ is a given function representing the rate of fluid jump between the two pore scales. Provided that $\beta_{1} \neq 0$, we can solve for $\dot{\psi}$ and substitute into (33) to obtain

$$
\dot{\bar{p}}_{c}=\frac{\alpha_{1}}{\beta_{1}} \dot{\pi}-\left(\frac{\alpha_{1} \beta_{2}}{\beta_{1}}-\alpha_{2}\right) \dot{\lambda} .
$$

To determine the consistency parameter, we substitute the constitutive equation (31) and hardening law (35) into the consistency condition (32) and solve for $\dot{\lambda}$. The result reads

$$
\dot{\lambda}=\frac{1}{\chi}\left(\frac{\partial F}{\partial \overline{\boldsymbol{\tau}}}: \boldsymbol{\alpha}^{\mathrm{e}}: \boldsymbol{d}+\frac{\partial F}{\partial \bar{p}_{c}} \frac{\alpha_{1}}{\beta_{1}} \dot{\pi}\right),
$$

where

$$
\chi=\frac{\partial F}{\partial \overline{\boldsymbol{\tau}}}: \boldsymbol{\alpha}^{\mathrm{e}}: \frac{\partial G}{\partial \overline{\boldsymbol{\tau}}}+H, \quad H=\frac{\partial F}{\partial \bar{p}_{c}}\left(\alpha_{2}-\frac{\alpha_{1} \beta_{2}}{\beta_{1}}\right) .
$$

Substituting the consistency parameter back into the constitutive equation (31) and simplifying yields

$$
\dot{\bar{\tau}}=\boldsymbol{\alpha}^{\mathrm{ep}}: \boldsymbol{d}+\boldsymbol{\theta} \dot{\pi},
$$

where

$$
\boldsymbol{\alpha}^{\mathrm{ep}}=\boldsymbol{\alpha}^{\mathrm{e}}-\frac{1}{\chi} \boldsymbol{\alpha}^{\mathrm{e}}: \frac{\partial G}{\partial \overline{\boldsymbol{\tau}}} \otimes \frac{\partial F}{\partial \overline{\boldsymbol{\tau}}}: \boldsymbol{\alpha}^{\mathrm{e}}
$$

and

$$
\boldsymbol{\theta}=-\frac{1}{\chi} \frac{\partial F}{\partial \bar{p}_{c}} \frac{\alpha_{1}}{\beta_{1}} \boldsymbol{\alpha}^{\mathrm{e}}: \frac{\partial G}{\partial \overline{\boldsymbol{\tau}}} .
$$

This result indicates that the effective Kirchhoff stress is driven not only by the solid velocity gradient but also by the fluid pressure jump between the two pore scales, which in turn arises from the difference in the preconsolidation stresses between the macropores and micropores.

\section{Finite element formulation}

This section presents a $\boldsymbol{u} / p_{M} / p_{m}$ finite element formulation for porous materials with double porosity, where $\boldsymbol{u}=$ solid displacement vector, $p_{M}=$ macropore fluid pressure, and $p_{m}=$ micropore fluid pressure. The formulation follows the standard lines of hyromechanical coupling $[10,16,17,23,24,34$, $38,56,62,72,73,78,81]$, except that it now treats the micropore fraction $\psi$ as an internal variable related to the independent variables by the relevant constitutive law. The section concludes with a discussion of the stress-point integration algorithm accommodating the evolution of the pore fraction in the finite deformation range. 


\subsection{Conservation laws and strong form}

Before presenting the relevant conservation laws, we first recall some preliminary results pertinent to finite deformation analysis (see [70, 71]). The first Piola-Kirchhoff stress tensor is $\boldsymbol{P}=\boldsymbol{\tau} \cdot \boldsymbol{F}^{-\mathrm{T}}$ and the corresponding effective stress tensor is $\overline{\boldsymbol{P}}=\overline{\boldsymbol{\tau}} \cdot \boldsymbol{F}^{-\mathrm{T}}$. The effective stress equation in terms of these stress tensors takes the form

$$
\boldsymbol{P}=\overline{\boldsymbol{P}}-\bar{p} \boldsymbol{F}^{-\mathrm{T}}
$$

where $\bar{p}$ is the Kirchhoff mean fluid pressure defined in equation (13).

The total mass density is the sum of the partial mass densities,

$$
\rho=\rho^{s}+\rho^{M}+\rho^{m}
$$

The partial mass densities are given by

$$
\rho^{s}=\phi^{s} \rho_{s}, \quad \rho^{M}=\left(1-\phi^{s}\right)(1-\psi) \rho_{w}, \quad \rho^{m}=\left(1-\phi^{s}\right) \psi \rho_{w},
$$

where the intrinsic mass densities $\rho_{s}$ and $\rho_{w}$ are assumed constant. The pullback mass densities are

$$
\rho_{0}=J \rho=\rho_{0}^{s}+\rho_{0}^{M}+\rho_{0}^{m} .
$$

We recall that $\dot{\rho}_{0}^{s}=\frac{\dot{J}}{J \rho^{s}}=0$ if the solid does not exchange mass with the fluid, see equation (5). Thus,

$$
\begin{aligned}
& \dot{\rho}_{0}^{M}=\dot{\cdot} \overline{J \rho^{M}}=(1-\psi) \dot{J} \rho_{w}-J\left(1-\phi^{s}\right) \dot{\psi} \rho_{w} \\
& \dot{\rho}_{0}^{m}=\bar{\cdot} \dot{\rho}^{m}=\psi \dot{J} \rho_{w}+J\left(1-\phi^{s}\right) \dot{\psi} \rho_{w}
\end{aligned}
$$

The relative fluid velocities in the macropores and micropores are defined with respect to the solid velocity $\boldsymbol{v}$ and take the form

$$
\tilde{\boldsymbol{v}}_{M}=\boldsymbol{v}_{M}-\boldsymbol{v}, \quad \tilde{\boldsymbol{v}}_{m}=\boldsymbol{v}_{m}-\boldsymbol{v}
$$

where $\boldsymbol{v}_{M}$ and $\boldsymbol{v}_{m}$ are the fluid velocities in the macropores and micropores, respectively. These relative velocities give rise to fluid mass fluxes

$$
\boldsymbol{q}_{M}=\rho^{M} \tilde{\boldsymbol{v}}_{M}, \quad \boldsymbol{q}_{m}=\rho^{m} \tilde{\boldsymbol{v}}_{m},
$$

with Piola transforms

$$
\boldsymbol{Q}_{M}=J \boldsymbol{F}^{-1} \cdot \boldsymbol{q}_{M}, \quad \boldsymbol{Q}_{m}=J \boldsymbol{F}^{-1} \cdot \boldsymbol{q}_{m} .
$$

We now consider a fluid-saturated body $\mathcal{B}$ with boundary $\partial \mathcal{B}$ in the reference configuration, and assume that the solid matrix possesses two porosity scales. The boundary $\partial \mathcal{B}$ admits the decomposition 


$$
\partial \mathcal{B}=\overline{\partial \mathcal{B}_{u} \cup \partial \mathcal{B}_{t}}, \quad \varnothing=\partial \mathcal{B}_{u} \cap \partial \mathcal{B}_{t},
$$

where $\partial \mathcal{B}_{u}$ and $\partial \mathcal{B}_{t}$ are Dirichlet and Neumann boundaries with displacement and tractions prescribed. For quasistatic loading the balance of linear momentum takes the form [13]

$$
\operatorname{DIV}(\boldsymbol{P})+\rho_{0} \boldsymbol{G}=c_{0}\left(\tilde{\boldsymbol{v}}_{m}-\tilde{\boldsymbol{v}}_{M}\right) \text { in } \mathcal{B},
$$

where DIV is the Lagrangian divergence operator, $\boldsymbol{G}$ is the gravity acceleration vector, and $c_{0}=J c$ is the pullback fluid mass transfer coefficient, with $c$ being the value in the current configuration, reflecting transfer of fluid mass into or from the micropores. The relevant boundary and initial conditions are $\boldsymbol{u}=\boldsymbol{u}^{*}$ on $\partial \mathcal{B}_{u}, \boldsymbol{P} \cdot \boldsymbol{N}=\boldsymbol{t}$ on $\partial \mathcal{B}_{t}$, and $\boldsymbol{u}(\boldsymbol{X})=\boldsymbol{u}_{0}(\boldsymbol{X})$ at time $t=0$ and for all $\boldsymbol{X} \in \mathcal{B}$.

We also consider the following decomposition of $\partial \mathcal{B}$

$$
\partial \mathcal{B}=\overline{\partial \mathcal{B}_{p_{M}} \cup \partial \mathcal{B}_{q_{M}}}, \quad \varnothing=\partial \mathcal{B}_{p_{M}} \cap \partial \mathcal{B}_{q_{M}}
$$

for the macropores, and

$$
\partial \mathcal{B}=\overline{\partial \mathcal{B}_{p_{m}} \cup \partial \mathcal{B}_{q_{m}}}, \quad \varnothing=\partial \mathcal{B}_{p_{m}} \cap \partial \mathcal{B}_{q_{m}}
$$

for the micropores, where $p$ and $q$ are pressure and flux boundary conditions, respectively. The fluid mass conservation equations in the macropores and micropores take the form [13]

$$
\begin{aligned}
\dot{\rho}_{0}^{M}+\operatorname{DIV}\left(\boldsymbol{Q}_{M}\right) & =-c_{0} & \text { in } \mathcal{B} \\
\dot{\rho}_{0}^{m}+\operatorname{DIV}\left(\boldsymbol{Q}_{m}\right) & =c_{0} & \text { in } \mathcal{B} .
\end{aligned}
$$

The boundary conditions are as follows: $p_{M}=p_{M}^{*}$ on $\partial \mathcal{B}_{p_{M}}, p_{m}=p_{m}^{*}$ on $\partial \mathcal{B}_{p_{m}}, \boldsymbol{Q}_{M} \cdot \boldsymbol{N}=Q_{M}$ on $\partial \mathcal{B}_{q_{M}}$, and $\boldsymbol{Q}_{m} \cdot \boldsymbol{N}=Q_{m}$ on $\partial \mathcal{B}_{q_{m}}$. The initial conditions are: $p_{M}(\boldsymbol{X})=p_{M 0}(\boldsymbol{X})$ and $p_{m}(\boldsymbol{X})=p_{m 0}(\boldsymbol{X})$ at time $t=0$ and for all $\boldsymbol{X} \in \mathcal{B}$.

\subsection{Variational equations}

To develop the weak form, we need the space of configurations denoted by

$$
\mathscr{C}_{u}=\left\{\boldsymbol{u}: \mathcal{B} \rightarrow \mathbb{R}^{n_{s d}} \mid u_{i} \in H^{1}, \boldsymbol{u}=\boldsymbol{u}^{*} \text { on } \partial \mathcal{B}_{u}\right\}
$$

and the space of variations

$$
\mathscr{V}_{u}=\left\{\boldsymbol{\eta}: \mathcal{B} \rightarrow \mathbb{R}^{n_{s d}} \mid \eta_{i} \in H^{1}, \boldsymbol{\eta}=\mathbf{0} \text { on } \partial \mathcal{B}_{u}\right\},
$$

where $H^{1}$ is the Sobolev space of functions of degree one. The variational form of linear momentum balance is given by

$$
\int_{\mathcal{B}}\left(\operatorname{GRAD} \boldsymbol{\eta}: \boldsymbol{P}-\rho_{0} \boldsymbol{\eta} \cdot \boldsymbol{G}+c_{0}\left(\tilde{\boldsymbol{v}}_{m}-\tilde{\boldsymbol{v}}_{M}\right)\right) \mathrm{d} V-\int_{\partial \mathcal{B}_{t}} \boldsymbol{\eta} \cdot \boldsymbol{t} \mathrm{d} A=0
$$


for all $\boldsymbol{\eta} \in \mathscr{V}_{u}$.

Next, we consider the spaces of pore pressures as

$$
\mathscr{C}_{p_{i}}=\left\{p_{i}: \mathcal{B} \rightarrow \mathbb{R} \mid p_{i} \in H^{1}, p_{i}=p_{i}^{*} \text { on } \partial \mathcal{B}_{p_{i}}\right\}, \quad i=M, m .
$$

and the spaces of variations

$$
\mathscr{V}_{\phi_{i}}=\left\{\phi_{i}: \mathcal{B} \rightarrow \mathbb{R} \mid \phi_{i} \in H^{1}, \phi_{i}=0 \text { on } \partial \mathcal{B}_{p_{i}}\right\}, \quad i=M, m .
$$

The variational form of balance of fluid mass takes the form (no sum on $i$ )

$$
\int_{\mathcal{B}} \phi_{i}\left(\dot{\rho}_{0}^{i}+a_{0}\right) \mathrm{d} V-\int_{\mathcal{B}} \operatorname{GRAD} \phi^{i} \cdot \boldsymbol{Q}_{i} \mathrm{~d} V+\int_{\partial \mathcal{B}_{q_{i}}} \phi^{i} Q_{i} \mathrm{~d} A=0
$$

for all $\phi_{i} \in \mathscr{V}_{\phi_{i}}$, where

$$
a_{0}=\left\{\begin{aligned}
c_{0}, & \text { if } i=M, \\
-c_{0}, & \text { if } i=m .
\end{aligned}\right.
$$

Alternatively, the variational equation for the balance of linear momentum can be written in terms of the symmetric Kirchhoff effective stress tensor as

$$
\begin{aligned}
\int_{\mathcal{B}} \boldsymbol{\nabla}^{s} \boldsymbol{\eta}: \overline{\boldsymbol{\tau}} \mathrm{d} V- & \int_{\mathcal{B}} \boldsymbol{\nabla} \cdot \boldsymbol{\eta} \bar{p} \mathrm{~d} V-\int_{\mathcal{B}} \rho_{0} \boldsymbol{\eta} \cdot \boldsymbol{G} \mathrm{d} V \\
& +\int_{\mathcal{B}} c_{0}\left(\tilde{\boldsymbol{v}}_{m}-\tilde{\boldsymbol{v}}_{M}\right) \mathrm{d} V=\int_{\partial \mathcal{B}_{t}} \boldsymbol{\eta} \cdot \boldsymbol{t} \mathrm{d} A
\end{aligned}
$$

for all $\boldsymbol{\eta} \in \mathscr{V}_{u}$. As for the variational equation for fluid mass conservation, we can use the backward implicit scheme to write the variational equation in the time-integrated form

$$
\begin{aligned}
\int_{\mathcal{B}} \phi_{i}\left(\rho_{0}^{i}-\rho_{0 n}^{i}\right) \mathrm{d} V-\Delta t \rho_{w} \int_{\mathcal{B}} \nabla \phi^{i} \cdot J \tilde{\boldsymbol{v}}_{i} \mathrm{~d} V \\
-\Delta t \int_{\mathcal{B}} \phi^{i} a_{0} \mathrm{~d} V=-\Delta t \int_{\partial \mathcal{B}_{q_{i}}} \phi^{i} Q_{i} \mathrm{~d} A,
\end{aligned}
$$

for all $\phi_{i} \in \mathscr{V}_{\phi_{i}}$, where $\Delta t=t-t_{n}$ is the step size.

\subsection{Linearized variational equations}

Before developing the linearized variation equations, we first introduce Darcy's law so as to reduce the finite element equations to $\boldsymbol{u} / p_{M} / p_{m}$ form. For double porosity media, Darcy's law takes the form

$$
J \tilde{\boldsymbol{v}}_{M}=-\boldsymbol{K}_{M} \cdot \boldsymbol{\nabla} \mathcal{U}_{M}, \quad J \tilde{\boldsymbol{v}}_{m}=-\boldsymbol{K}_{m} \cdot \boldsymbol{\nabla} \mathcal{U}_{m},
$$

where $\mathcal{U}_{M}$ and $\mathcal{U}_{m}$ are the fluid potentials in the macropores and micropores, respectively, given by 


$$
\mathcal{U}_{M}=\frac{p_{M}}{\rho_{w} g}+z, \quad \mathcal{U}_{m}=\frac{p_{m}}{\rho_{w} g}+z,
$$

where $z$ is the elevation axis and $g$ is the gravity acceleration constant. Assuming isotropy in permeability, the pullback permeability tensor $\boldsymbol{K}$ can be expressed in terms of effective grain diameter $D$ and jacobian $J$ through an equation of the form

$$
\boldsymbol{K}_{M}=K\left(D_{M}, J\right) \mathbf{1}, \quad \boldsymbol{K}_{m}=K\left(D_{m}, J\right) \mathbf{1}
$$

where $D_{M}$ is the effective diameter of the aggregates and $D_{m}$ is the effective diameter of the grains in the micropores. An example of an empirical expression for the scalar function $K(D, J)$ is given by the well-known Kozeny-Carman equation [8]

$$
K(D, J)=J k(D, J), \quad k(D, J)=\frac{\rho_{w} g}{\mu_{w}} \frac{D^{2}}{180} \frac{\left(J-\phi_{0}^{s}\right)^{3}}{J\left(\phi_{0}^{s}\right)^{2}},
$$

where $\mu_{w}$ is the dynamic viscosity of water and $\phi_{0}^{s}$ is the volume fraction of the solid when $J=1$. Alternatively, the Kozeny-Carman expression can be written in normalized form relative to the initial value of hydraulic conductivity and evolving according to $J$ in a fashion similar to the equation above. In the examples discussed in this paper, the evolutions of hydraulic conductivities are expressed in terms of initial values $k_{M 0}$ and $k_{m 0}$.

A second constitutive law leading to the $\boldsymbol{u} / p_{M} / p_{m}$ formulation determines the mass transfer term $c=c_{0} / J$ (see equation (51)) in terms of the fluid pressure jump. A vast number of studies have advanced constitutive relations for this term (see e.g., [30, 33, 39, 47, 63, 80]). Here, we adopt the constitutive law used by Choo et al. [24] of the form

$$
c=\frac{\bar{\alpha}}{\mu_{w}}\left(p_{M}-p_{m}\right),
$$

where $\bar{\alpha}$ is a dimensionless parameter that depends on the characteristics of the interface between the macropores and micropores, such as permeability, spacing, and shape. This parameter can be determined either by an equation that takes permeability and pore geometry into account (e.g. [33]), or by inversion of test data (e.g. [76]).

We now consider a constitutive equation for the effective Kirchhoff stress of the form

$$
\overline{\boldsymbol{\tau}}=\overline{\boldsymbol{\tau}}\left(\boldsymbol{u}, p_{M}, p_{m}\right) .
$$

Strictly speaking, $\overline{\boldsymbol{\tau}}$ is also a function of the pore fraction $\psi$, but this variable also depends on the independent variables $\boldsymbol{u}, p_{M}$, and $p_{m}$. Linearizing this stress tensor yields

$$
\delta \overline{\boldsymbol{\tau}}=\boldsymbol{\alpha}: \nabla \delta \boldsymbol{u}+\boldsymbol{a}_{M} \delta p_{M}+\boldsymbol{a}_{m} \delta p_{m},
$$


where $\boldsymbol{\alpha}$ is a tangential moduli tensor discussed in the next section, and $\boldsymbol{a}_{i}=\partial \overline{\boldsymbol{\tau}} / \partial p_{i}$ for $i=M, m$. Furthermore, from equation (44) the pullback total mass density has the linearization

$$
\delta \rho_{0}=\delta \rho_{0}^{M}+\delta \rho_{0}^{m}=\rho_{w} \delta J,
$$

where $\delta J=J \boldsymbol{\nabla} \cdot \delta \boldsymbol{u}$. This results in the following linearized variational equation for balance of momentum

$$
\begin{array}{r}
\int_{\mathcal{B}} \boldsymbol{\nabla} \boldsymbol{\eta}: \mathbf{a}: \boldsymbol{\nabla} \delta \boldsymbol{u} \mathrm{d} V+\int_{\mathcal{B}} \boldsymbol{\eta}:\left(\boldsymbol{a}_{M} \delta p_{M}+\boldsymbol{a}_{m} \delta p_{m}\right) \mathrm{d} V \\
-\int_{\mathcal{B}}(\delta(\boldsymbol{\nabla} \cdot \boldsymbol{\eta}) \bar{p}+\boldsymbol{\nabla} \cdot \boldsymbol{\eta} \delta \bar{p}) \mathrm{d} V-\int_{\mathcal{B}} \delta \rho_{0} \boldsymbol{\eta} \cdot \boldsymbol{G} \mathrm{d} V \\
\quad+\delta \int_{\mathcal{B}} c \boldsymbol{\eta} \cdot\left(J \tilde{\boldsymbol{v}}_{m}-J \tilde{\boldsymbol{v}}_{M}\right) \mathrm{d} V=\int_{\partial \mathcal{B}} \boldsymbol{\eta} \cdot \delta \boldsymbol{t} \mathrm{d} A
\end{array}
$$

where $\mathbf{a}=\boldsymbol{\alpha}-\overline{\boldsymbol{\tau}} \ominus \mathbf{1}, \delta(\boldsymbol{\nabla} \cdot \boldsymbol{\eta})=-\boldsymbol{\nabla} \boldsymbol{\eta}: \delta \boldsymbol{u} \boldsymbol{\nabla}$, and $c=c_{0} / J$, see the discussion surrounding equation (51). The stress term in the tangent moduli has components $(\overline{\boldsymbol{\tau}} \ominus \mathbf{1})_{i j k l}=\bar{\tau}_{i l} \delta_{j k}$.

Eliminating the relative fluid velocities using Darcy's law, and linearizing the associated volume integral, yields

$$
\begin{aligned}
\delta \int_{\mathcal{B}} c \boldsymbol{\eta} \cdot\left(J \tilde{\boldsymbol{v}}_{m}-J \tilde{\boldsymbol{v}}_{M}\right) \mathrm{d} & =\int_{\mathcal{B}} c \boldsymbol{\eta} \cdot \delta\left(\boldsymbol{K}_{M} \cdot \boldsymbol{\nabla} \mathcal{U}_{M}-\boldsymbol{K}_{m} \cdot \nabla \mathcal{U}_{m}\right) \mathrm{d} V \\
& -\int_{\mathcal{B}} \delta c \boldsymbol{\eta} \cdot\left(\boldsymbol{K}_{M} \cdot \boldsymbol{\nabla} \mathcal{U}_{M}-\boldsymbol{K}_{m} \cdot \nabla \mathcal{U}_{m}\right) \mathrm{d} V
\end{aligned}
$$

Disregarding the subscripts, we note that

$$
\delta(\boldsymbol{K} \cdot \nabla \mathcal{U})=\delta \boldsymbol{K} \cdot \boldsymbol{\nabla} \mathcal{U}+\boldsymbol{K} \cdot \delta(\nabla \mathcal{U})
$$

where

$$
\delta \boldsymbol{K}=\left(K^{\prime}(J) \delta J\right) \mathbf{1}, \quad \delta(\boldsymbol{\nabla U})=\boldsymbol{\nabla} \delta p-\boldsymbol{\nabla} p \cdot \boldsymbol{\nabla} \delta \boldsymbol{u} .
$$

Furthermore,

$$
\delta c=\frac{\bar{\alpha}}{\mu_{w}}\left(\delta p_{M}-\delta p_{m}\right) .
$$

See Song and Borja [70] for further details on these derivatives.

Next we consider the linearization of the time-integrated variational equation for the balance of fluid mass. For pore scale $i=M, m$, we have

$$
\begin{aligned}
\int_{\mathcal{B}} \phi_{i} \delta \rho_{0}^{i} \mathrm{~d} V & -\Delta t \rho_{w} \delta\left(\int_{\mathcal{B}} \nabla \phi^{i} \cdot \boldsymbol{K}_{i} \cdot \nabla \mathcal{U} \mathrm{d} V\right) \\
& -\Delta t \int_{\mathcal{B}} \phi^{i} \delta a_{0} \mathrm{~d} V=-\Delta t \int_{\partial \mathcal{B}_{q_{i}}} \phi^{i} \delta Q_{i} \mathrm{~d} A,
\end{aligned}
$$

where $\delta a_{0}$ follows from (78) and $\delta \rho_{0}^{i}$ follows from (45) and (46). The flux term has the linearization 


$$
\begin{aligned}
\delta\left(\int_{\mathcal{B}} \boldsymbol{\nabla} \phi^{i} \cdot \boldsymbol{K}_{i} \cdot \nabla \mathcal{U} \mathrm{d} V\right)=\int_{\mathcal{B}} \delta\left(\nabla \phi^{i}\right) \cdot \boldsymbol{K}_{i} \cdot \nabla \mathcal{U} \mathrm{d} V \\
\quad+\int_{\mathcal{B}} \boldsymbol{\nabla} \phi^{i} \cdot \boldsymbol{K}_{i} \cdot \delta(\boldsymbol{\nabla} \mathcal{U}) \mathrm{d} V+\int_{\mathcal{B}} \boldsymbol{\nabla} \phi^{i} \cdot \delta \boldsymbol{K}_{i} \cdot \boldsymbol{\nabla} \mathcal{U} \mathrm{d} V,
\end{aligned}
$$

where

$$
\delta\left(\boldsymbol{\nabla} \phi^{i}\right)=-\boldsymbol{\nabla} \phi^{i} \cdot \boldsymbol{\nabla} \delta \boldsymbol{u} .
$$

It remains to determine the tangent operators $\boldsymbol{\alpha}, \boldsymbol{a}_{M}$ and $\boldsymbol{a}_{m}$ in equation (74); these are developed in the next section.

\section{Discrete formulation}

This section describes the discrete evolution of the pore fraction $\psi$, which measures the internal structure in a double-porosity material, as well as its impact on the effective stress tensor $\overline{\boldsymbol{\tau}}$. For constitutive modeling of the solid material, we employ the classic three-invariant hyperelasto-plasticity and solve the local unknowns in principal elastic strain space, see [12].

\subsection{Stress-point integration}

We recall the classic stress-point integration for finite deformation hyperelastoplasticity consisting of an elastic predictor for the elastic left CauchyGreen deformation tensor $\boldsymbol{b}^{\mathrm{e}}$, followed by a plastic corrector in elastic logarithmic principal strains [12]. The elastic predictor is calculated from the relative deformation gradient $\boldsymbol{f}=\partial \boldsymbol{x} / \partial \boldsymbol{x}_{n}$ and the current value of the elastic left Cauchy-Green deformation tensor $\boldsymbol{b}_{n}^{\mathrm{e}}$, after which a spectral decomposition is performed, i.e.

$$
\boldsymbol{b}^{\mathrm{e} \operatorname{tr}}=\boldsymbol{f} \cdot \boldsymbol{b}_{n}^{\mathrm{e}} \cdot \boldsymbol{f}^{\boldsymbol{\top}}=\sum_{A=1}^{3}\left(\lambda_{A}^{\mathrm{etr}}\right)^{2} \boldsymbol{m}^{(A)},
$$

where $\lambda_{A}^{\mathrm{e} t r}$ is the trial elastic principal stretch with an associated principal direction $\boldsymbol{n}^{(A)}$ and spectral direction $\boldsymbol{m}^{(A)}=\boldsymbol{n}^{(A)} \otimes \boldsymbol{n}^{(A)}$. By coaxiality of $\boldsymbol{b}^{\mathrm{etr}}$ and $\overline{\boldsymbol{\tau}}$, we have

$$
\overline{\boldsymbol{\tau}}=\sum_{A=1}^{3} \bar{\tau}_{A} \boldsymbol{m}^{(A)}
$$

where $\bar{\tau}_{A}$ is a principal value of the effective Kirchhoff stress tensor obtained from the hyperelastic constitutive equation

$$
\bar{\tau}_{A}=\frac{\partial \Psi^{\mathrm{e}}}{\partial \varepsilon_{A}^{\mathrm{e}}}, \quad \varepsilon_{A}^{\mathrm{e}}=\log \left(\lambda_{A}^{\mathrm{e}}\right),
$$

in which $\Psi^{\mathrm{e}}$ is the stored energy function and $\varepsilon_{A}^{\mathrm{e}}$ is the elastic logarithmic

principal strain determined from the logarithm of the elastic principal stretch 
$\lambda_{A}^{\mathrm{e}}$. The stress-point integration algorithm entails the determination of the evolution of $\varepsilon_{A}^{\mathrm{e}}$ along with those of the internal state variables including the pore fraction $\psi$.

The local stress-point integration is as follows. Given the following starting values at $t_{n}:\left(\bar{\tau}_{A}\right)_{n}$ for $A=1,2,3 ;\left(p_{M}\right)_{n},\left(p_{m}\right)_{n}$, micropore fraction $\psi_{n}$, and preconsolidation pressures $\left(p_{c M}\right)_{n}$ and $\left(p_{c n}\right)_{n}$; and given increments $\Delta \boldsymbol{u}$, $\Delta p_{M}$, and $\Delta p_{m}$; find the principal stresses $\bar{\tau}_{A} \equiv\left(\bar{\tau}_{A}\right)_{n+1}$ for $A=1,2,3$, and micropore fraction $\psi \equiv \psi_{n+1}$. To accommodate nonlinear hyperelasticity we use the notion of residuals and perform a return mapping in principal logarithmic elastic strain space as follows:

$$
r_{A}=\varepsilon_{A}^{\mathrm{e}}-\varepsilon_{A}^{\mathrm{e} t \mathrm{r}}+\Delta \lambda \frac{\partial F}{\partial \bar{\tau}_{A}} \rightarrow 0, \quad A=1,2,3,
$$

where $\varepsilon_{A}^{\mathrm{etr}}=\log \left(\lambda_{A}^{\mathrm{e}} \mathrm{tr}\right)$ and $\Delta \lambda$ is the incremental plastic consistency parameter. Next, we impose the yield criterion

$$
r_{4}=F\left(\bar{\tau}_{1}, \bar{\tau}_{2}, \bar{\tau}_{3}, \bar{p}_{c}\right) \rightarrow 0,
$$

where $\bar{p}_{c}$ is the mean preconsolidation pressure defined from the hardening law

$$
r_{5}=\bar{p}_{c}-\psi p_{c m}\left(\psi, \sum_{A=1}^{3} \varepsilon_{A}^{\mathrm{e}}\right)-(1-\psi) p_{c M}\left(\psi, \sum_{A=1}^{3} \varepsilon_{A}^{\mathrm{etr}}\right) \rightarrow 0 .
$$

The final residual term is the thermodynamic restriction

$$
r_{6}=p_{c m}\left(\psi, \sum_{A=1}^{3} \varepsilon_{A}^{\mathrm{e}}, \sum_{A=1}^{3} \varepsilon_{A}^{\mathrm{etr}}\right)-p_{c M}\left(\psi, \sum_{A=1}^{3} \varepsilon_{A}^{\mathrm{etr}}\right)+\left(p_{M}-p_{m}\right) \rightarrow 0,
$$

where $p_{M}=\left(p_{M}\right)_{n}+\Delta p_{M}$ and $p_{m}=\left(p_{m}\right)_{n}+\Delta p_{m}$. It must be noted that in the above equations, the evolutions of $\varepsilon_{A}^{\mathrm{etr}}$ (for $A=1,2,3$ ), $p_{M}$, and $p_{m}$ are all given, and thus, we have a total of six residual equations in six unknowns, namely, $\varepsilon_{A}^{\mathrm{e}}(A=1,2,3), \Delta \lambda, \bar{p}_{c}$, and $\psi$.

The six unknowns are solved by a local Newton iteration. Setting

$$
\boldsymbol{r}=\left\{\begin{array}{l}
r_{1} \\
r_{2} \\
r_{3} \\
r_{4} \\
r_{5} \\
r_{6}
\end{array}\right\}, \quad \boldsymbol{x}=\left\{\begin{array}{c}
\varepsilon_{1}^{\mathrm{e}} \\
\varepsilon_{2}^{\mathrm{e}} \\
\varepsilon_{3}^{\mathrm{e}} \\
\Delta \lambda \\
\bar{p}_{c} \\
\psi
\end{array}\right\}, \quad \boldsymbol{A}=\boldsymbol{r}^{\prime}(\boldsymbol{x}),
$$

the problem is then to find the solution $\boldsymbol{x}^{*}$ such that $\boldsymbol{r}=\mathbf{0}$. The elements of the local tangent operator $\boldsymbol{r}^{\prime}(\boldsymbol{x})$ are developed in Appendix A. 


\subsection{Tangent operators}

In this section we develop expressions for the global tangent operators accommodating the variation of the pore fraction $\psi$. The key aspect to obtaining these tangent operators is the consistent linearization of the local residual vector $\boldsymbol{r}$ in (89) through the local tangent operator $\boldsymbol{A}$, which is available in closed form as shown in the Appendix A.

Consider the functional representation of $\overline{\boldsymbol{\tau}}$ in terms of $\boldsymbol{u}, p_{M}$, and $p_{m}$ as indicated in (71), and its spectral representation shown in (83). We note that the spectral directions of $\overline{\boldsymbol{\tau}}$ depend on $\boldsymbol{u}$ alone through $\boldsymbol{b}^{\mathrm{e}}$, whereas its principal values depend not only on $\varepsilon_{A}^{\mathrm{etr}}$ but also on $p_{M}$ and $p_{m}$, all of them through the principal elastic logarithmic strains $\varepsilon_{A}^{\mathrm{e}}$ for $A=1,2,3$. Therefore, we can write the variation of $\bar{\tau}$ as

$$
\delta \overline{\boldsymbol{\tau}}=\sum_{A=1}^{3}\left(\delta \bar{\tau}_{A} \boldsymbol{m}^{(A)}+\bar{\tau}_{A} \delta \boldsymbol{m}^{(A)}\right)
$$

where

$$
\begin{aligned}
\delta \bar{\tau}_{A} & =\sum_{C=1}^{3} \frac{\partial \bar{\tau}_{A}}{\partial \varepsilon_{C}^{\mathrm{e}}}\left(\sum_{B=1}^{3} \frac{\partial \varepsilon_{C}^{\mathrm{e}}}{\partial \varepsilon_{B}^{\mathrm{etr}}} \delta \varepsilon_{B}^{\mathrm{e} t \mathrm{tr}}+\frac{\partial \varepsilon_{C}^{\mathrm{e}}}{\partial p_{M}} \delta p_{M}+\frac{\partial \varepsilon_{C}^{\mathrm{e}}}{\partial p_{m}} \delta p_{m}\right) \\
& =\sum_{B=1}^{3} a_{A B} \delta \varepsilon_{B}^{\mathrm{e} t \mathrm{tr}}+\frac{\partial \bar{\tau}_{A}}{\partial p_{M}} \delta p_{M}+\frac{\partial \bar{\tau}_{A}}{\partial p_{m}} \delta p_{m},
\end{aligned}
$$

and

$$
\begin{array}{rlrl}
a_{A B} & =\sum_{C=1}^{3} a_{A C}^{\mathrm{e}} \frac{\partial \varepsilon_{C}^{\mathrm{e}}}{\partial \varepsilon_{B}^{\mathrm{e} t r}}, & \frac{\partial \bar{\tau}_{A}}{\partial p_{M}} & =\sum_{C=1}^{3} a_{A C}^{\mathrm{e}} \frac{\partial \varepsilon_{C}^{\mathrm{e}}}{\partial p_{M}} \\
\frac{\partial \bar{\tau}_{A}}{\partial p_{m}}=\sum_{C=1}^{3} a_{A C}^{\mathrm{e}} \frac{\partial \varepsilon_{C}^{\mathrm{e}}}{\partial p_{m}}, & a_{A C}^{\mathrm{e}}=\frac{\partial \bar{\tau}_{A}}{\partial \varepsilon_{C}^{\mathrm{e}}}=\frac{\partial^{2} \Psi^{\mathrm{e}}}{\partial \varepsilon_{A}^{\mathrm{e}} \partial \varepsilon_{C}^{\mathrm{e}}} .
\end{array}
$$

Furthermore, the spin of $\overline{\boldsymbol{\tau}}$ is the same as the spin of $\boldsymbol{b}^{\mathrm{e} t r}$, and so,

$$
\sum_{A=1}^{3} \bar{\tau}_{A} \delta \boldsymbol{m}^{(A)}=\sum_{A=1}^{3} \sum_{B \neq A} \omega_{A B}\left(\bar{\tau}_{B}-\bar{\tau}_{A}\right) \boldsymbol{m}^{(A B)}
$$

where $\omega_{A B}$ is the spin of $\boldsymbol{b}^{\mathrm{etr}}$, and $\boldsymbol{m}^{(A B)}=\boldsymbol{n}^{(A)} \otimes \boldsymbol{n}^{(B)}$.

The relevant tangent operators of interest, defined in (72), then take the following forms 


$$
\begin{aligned}
\boldsymbol{\alpha} & =\sum_{A=1}^{3} \sum_{B=1}^{3} a_{A B} \boldsymbol{m}^{(A)} \otimes \boldsymbol{m}^{(B)}+\sum_{A=1}^{3} \sum_{B \neq A} \frac{\bar{\tau}_{B}-\bar{\tau}_{A}}{\left(\lambda_{B}^{\mathrm{etr}}\right)^{2}-\left(\lambda_{A}^{\mathrm{etr}}\right)^{2}} \boldsymbol{\beta}_{A B}, \\
\boldsymbol{\beta}_{A B} & =\left(\lambda_{B}^{\mathrm{e} \text { tr }}\right)^{2} \boldsymbol{m}^{(A B)} \otimes \boldsymbol{m}^{(A B)}+\left(\lambda_{A}^{\mathrm{etr}}\right)^{2} \boldsymbol{m}^{(A B)} \otimes \boldsymbol{m}^{(B A)} \\
\boldsymbol{a}_{M} & =\sum_{A=1}^{3} \frac{\partial \bar{\tau}_{A}}{\partial p_{M}} \boldsymbol{m}^{(A)}, \quad \boldsymbol{a}_{m}=\sum_{A=1}^{3} \frac{\partial \bar{\tau}_{A}}{\partial p_{m}} \boldsymbol{m}^{(A)} .
\end{aligned}
$$

Complete development of the tangent operators relies on closed-form expressions for the derivatives of $\varepsilon_{A}^{\mathrm{e}}$.

Consider the converged residual equation of the local problem, $\boldsymbol{r}\left(\boldsymbol{x}^{*}\right)=\mathbf{0}$. We recall that this residual equation relies on the assumption that $\varepsilon_{A}^{\mathrm{etr}}, p_{M}$, and $p_{m}$ are all given. Thus, the residual vector may be viewed as a function of $\boldsymbol{z}$ as well, i.e., $\boldsymbol{r}=\boldsymbol{r}(\boldsymbol{z}, \boldsymbol{x})$, where

$$
\boldsymbol{z}:=\left\{\begin{array}{c}
\varepsilon_{1}^{\text {etr }} \\
\varepsilon_{2}^{\text {etr }} \\
\varepsilon_{3}^{\text {etr }} \\
p_{M} \\
p_{m}
\end{array}\right\} \quad \text { and }\left.\quad \frac{\partial \boldsymbol{r}}{\partial \boldsymbol{z}}\right|_{\boldsymbol{x}^{*}}+\left.\boldsymbol{A} \frac{\partial \boldsymbol{x}}{\partial \boldsymbol{z}}\right|_{\boldsymbol{x}^{*}}=\mathbf{0}
$$

In the foregoing equation, it is understood that $\boldsymbol{r}\left(\boldsymbol{z}, \boldsymbol{x}^{*}\right)=\mathbf{0}$, and thus, all the derivatives must be evaluated at $\boldsymbol{x}=\boldsymbol{x}^{*}$. This equation then yields the tangent matrix

$$
\boldsymbol{B}:=\frac{\partial \boldsymbol{x}}{\partial \boldsymbol{z}}=-\left.\boldsymbol{A}^{-1} \frac{\partial \boldsymbol{r}}{\partial \boldsymbol{z}}\right|_{\boldsymbol{x}} .
$$

The derivatives of interest can be obtained from the elements of the matrix $B$,

$$
\frac{\partial \varepsilon_{I}^{\mathrm{e}}}{\partial \varepsilon_{J}^{\mathrm{etr}}}=B_{I J}, \quad \frac{\partial \varepsilon_{I}^{\mathrm{e}}}{\partial p_{M}}=B_{I 4}, \quad \frac{\partial \varepsilon_{I}^{\mathrm{e}}}{\partial p_{m}}=B_{I 5},
$$

for $I, J=1,2,3$. Elements in the upper $3 \times 5$ block of the matrix $\boldsymbol{B}$ define all the required derivatives of $\varepsilon_{I}^{\mathrm{e}}$. The components of the tangent matrix $\partial \boldsymbol{r} / \partial \boldsymbol{z}$ are derived in Appendix B.

\subsection{Yield function}

We use a three-invariant plasticity model derived from enhancing the twoinvariant modified Cam-Clay theory. The three invariants of the effective Kirchhoff stress tensor $\overline{\boldsymbol{\tau}}$ are

$$
\tau_{m}=\frac{1}{3} \operatorname{tr}(\overline{\boldsymbol{\tau}}) \leq 0, \quad q=\sqrt{\frac{3}{2}}\|\boldsymbol{\xi}\|, \quad \frac{1}{\sqrt{6}} \cos 3 \theta=\frac{\operatorname{tr}\left(\boldsymbol{\xi}^{3}\right)}{\operatorname{tr}\left(\boldsymbol{\xi}^{2}\right)^{3 / 2}},
$$

where $\boldsymbol{\xi}=\overline{\boldsymbol{\tau}}-\tau_{m} \mathbf{1}$. The yield function is of the form (see [12]) 


$$
F=\zeta^{2} \frac{q^{2}}{M^{2}}+\tau_{m}\left(\tau_{m}-\bar{p}_{c}\right) \leq 0,
$$

where $M>0$ is the slope of the critical stress line, and $\zeta$ is the scaling function of the form $[5,37]$

$$
\zeta(\rho, \theta)=\frac{(1+\rho)+(1-\rho) \cos 3 \theta}{2 \rho} .
$$

The parameter $\rho$ should be in the range $7 / 9 \leq \rho \leq 1$ to guarantee smoothness and convexity. An associative flow rule is assumed throughout.

\section{Numerical examples}

The objectives of this section are twofold: (a) show that the proposed doubleporosity formulation can predict the experimental observations well, and (b) demonstrate the robust aspects of the algorithm in handling the concurrent transient fluid pressure dissipation and an evolving internal structure.

\subsection{Stress-point simulations}

As a first example, we conduct stress-point simulations of the compression of two aggregated soils, namely, Bioley silt and Corinth marl. The goal of the experiments was to investigate the compressibility characteristics of these two aggregated soils under 'drained' condition where $\bar{p}_{c}=p_{c M}=p_{c m}$. Because there is no transient pore pressure dissipation to consider, it suffices to treat the soil sample as a homogeneously deforming element and conduct stresspoint simulations on this element.

Koliji et al. [52] investigated the compressibility characteristics of an aggregated Bioley silt under a 1D compression test in which the lateral strain was held fixed while the sample was compressed vertically. The experiment entailed measuring the compressibility indices $c_{c}$ and $c_{r}$ for the reconstituted soil, as well as the compressibility index $c_{M}$ for the aggregated (structured) soil. Figure 3 shows the relevant experimental data for the structured soil with a preconsolidation pressure of $\bar{p}_{c}=-100 \mathrm{kPa}$, as well as for the reconstituted soil [52]. From these data, the following compressibility indices were determined: $c_{c}=0.085, c_{r}=0.009$, and $c_{M}=0.4$. Note that the macropores do not exhibit elastic deformations, and so all recoverable deformations can be attributed solely to the micropore deformations.

Koliji et al. [50] then used neutron tomography to investigate the evolution of the macropore void ratio $e_{M}$ as a function of the overall plastic volumetric strain for the same Bioley silt sample. Figure 4 shows the experimental data along with the evolution of $e_{M}$ predicted by the proposed constitutive formulation. Very good agreement can be observed. 


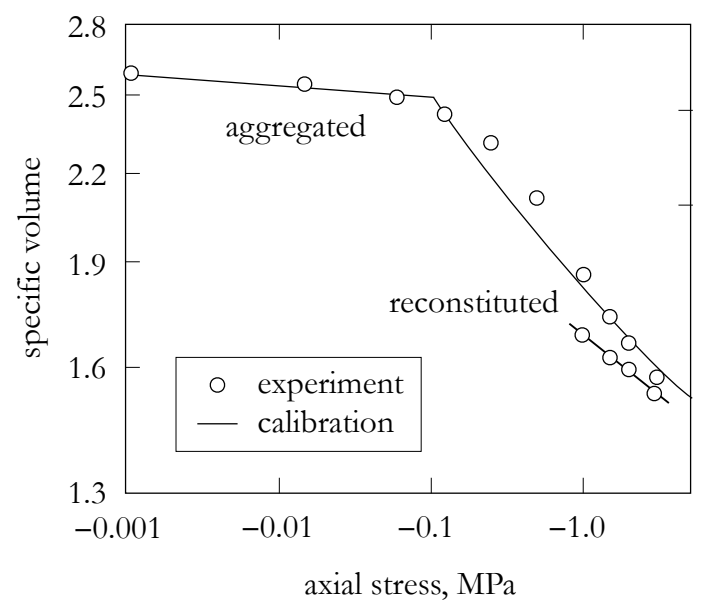

Fig. 3. Variation of specific volume with axial stress for aggregated and reconstituted Bioley silt. Experimental data from Koliji et al. [52].

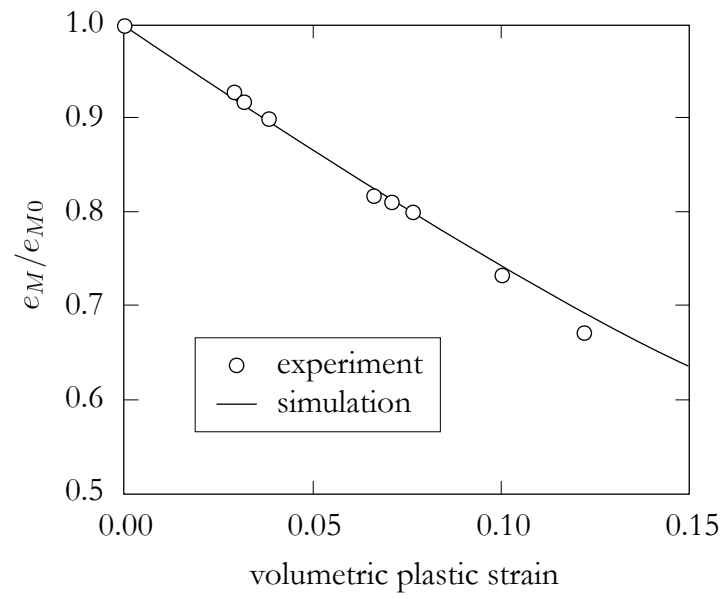

Fig. 4. Variation of macropore void ratio $e_{M}$ with volumetric plastic strain for Bioley silt. Initial value $e_{M 0}$ was calculated at a stress level equal to $\bar{p}_{c}=-100 \mathrm{kPa}$. Experimental data from Koliji et al. [52].

Next we consider a similar 1D compression test conducted by Anagnostopoulos et al. [4] on an aggregated sample of Corinth marl. Their testing program also included triaxial testing on the same soil in addition to $1 \mathrm{D}$ compression testing. Predicting the triaxial responses of this soil using parameters calibrated from the $1 \mathrm{D}$ compression test is a challenge since the two stress conditions are vastly different. The following discussions compare 
the triaxial responses predicted by the proposed constitutive framework with those reported previously in the literature.

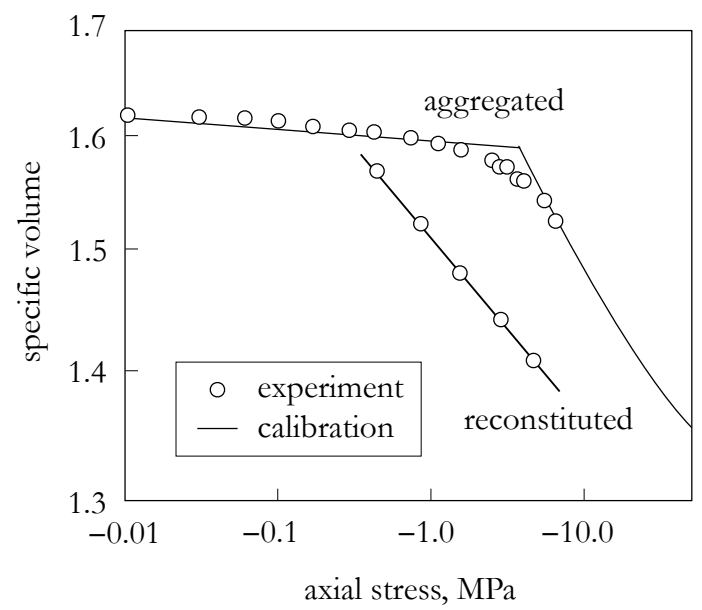

Fig. 5. Variation of specific volume with axial stress for aggregated and reconstituted Corinth marl. Experimental data from Anagnostopoulos et al. [4].

Figure 5 shows the experimental data from 1D compression tests on aggregated and reconstituted samples of Corinth marl [4]. From these data, the following compressibility indices have been determined for this soil: $c_{c}=0.046$, $c_{r}=0.004$, and $c_{M}=0.8$. Using these parameters, the triaxial test was then simulated as a stress-point problem and the results are shown in Figs. 6-9. We observe that the proposed constitutive formulation predicted both the deviator stress-axial strain and volumetric strain-axial strain responses reasonably well. In contrast, the model proposed by Liu and Carter [54] significantly overpredicted the deviator stress at a confining pressure of $4 \mathrm{MPa}$ (Fig. 6), and also overpredicted the volumetric strain at a confining pressure of $1.5 \mathrm{MPa}$ (Fig. 7). On the other hand, the constitutive framework proposed by Koliji et al. [48] captured the deviator stress-axial strain responses just as accurately as the proposed constitutive model (Fig. 8); however, their model underpredicted the volumetric compression at both confining pressures considerably (Fig. 9). For the two soils considered, the proposed constitutive formulation appears to adapt to other stress conditions more accurately.

\subsection{D consolidation with secondary compression}

Several investigators have argued that secondary compression in clays can be attributed in part to delayed dissipation of excess pore pressures in the micropores of multiple porosity media $[25,57,58]$. Considering that many 


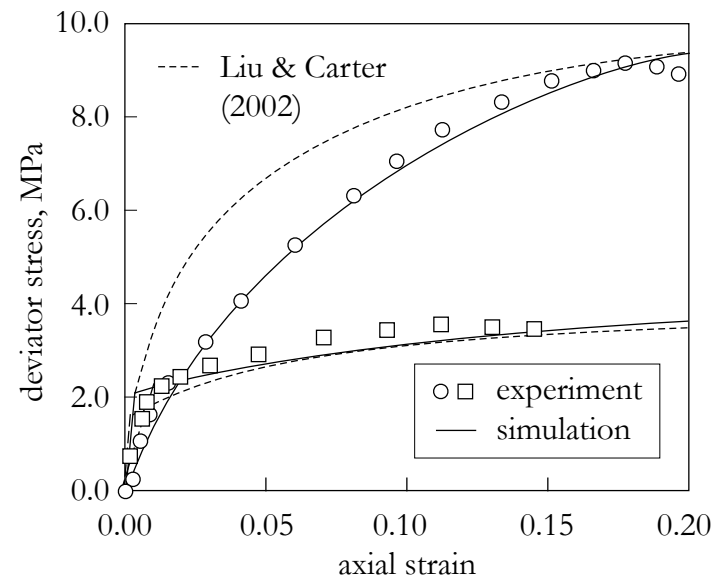

Fig. 6. Deviator stress versus axial strain for Corinth marl: comparison with Liu and Carter [54]. Experimental data from Anagnostopoulos et al. [4]: open circles are for test at confining pressure of $4 \mathrm{MPa}$, open squares at $1.5 \mathrm{MPa}$.

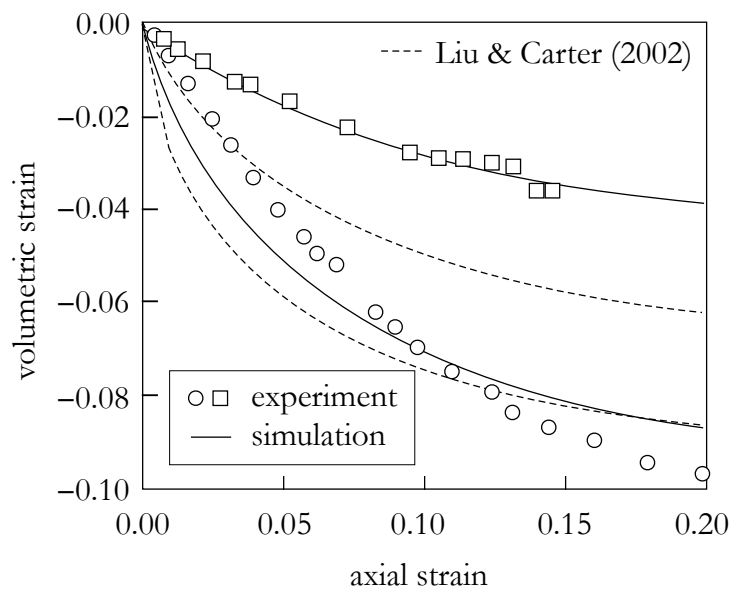

Fig. 7. Volumetric strain versus axial strain for Corinth marl: comparison with Liu and Carter [54]. Experimental data after Anagnostopoulos et al. [4], with open circles for test at confining pressure of $4 \mathrm{MPa}$, and open squares at $1.5 \mathrm{MPa}$.

aggregated soils such as peat show pronounced secondary consolidation, it is interesting to see if the proposed double-porosity formulation could indeed replicate such phenomena.

Figure 10 shows the finite element mesh considered in the simulation of one-dimensional consolidation process in a soil with double porosity. The do- 


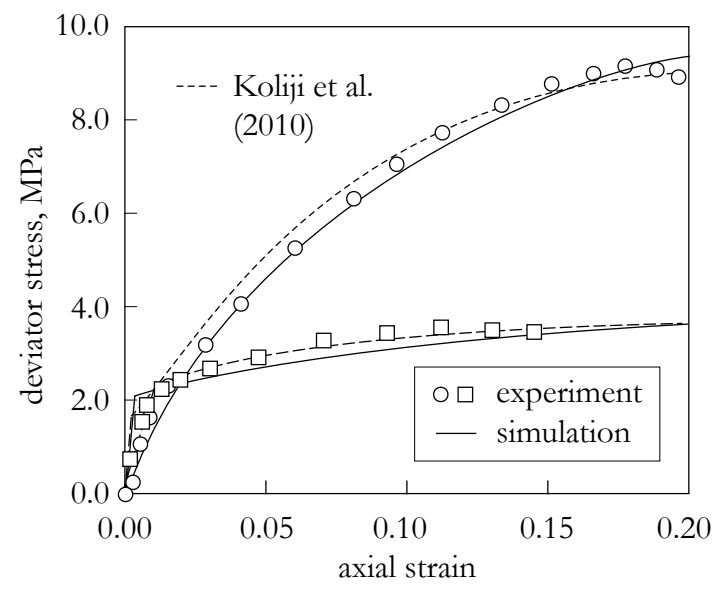

Fig. 8. Deviator stress versus axial strain responses for Corinth marl: comparison with Kolji et al. [48]. Experimental data from Anagnostopoulos et al. [4]: open circles are for test at confining pressure of $4 \mathrm{MPa}$, open squares at $1.5 \mathrm{MPa}$.

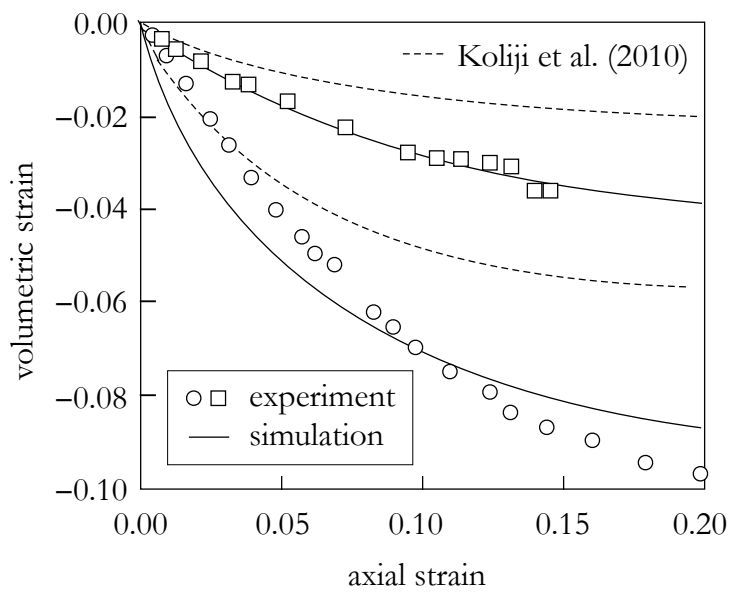

Fig. 9. Volumetric strain versus axial strain for Corinth marl: comparison with Kolji et al. [48]. Experimental data after Anagnostopoulos et al. [4], with open circles for test at confining pressure of $4 \mathrm{MPa}$, and open squares at $1.5 \mathrm{MPa}$.

main is $0.1 \mathrm{~m}$ wide and $1.0 \mathrm{~m}$ tall, and discretized into 20 quadrilateral mixed elements with biquadratic displacement interpolation of displacement and bilinear interpolation of the macropore and micropore pressures. The material parameters for the solid were assumed to be the same as those of Bioley silt presented in the previous example. The initial hydraulic conductivities for 
the macropores and micropores were taken as follows: $k_{M 0}=10^{-5} \mathrm{~m} / \mathrm{s}$ and $k_{m 0}=10^{-9} \mathrm{~m} / \mathrm{s}$. The mass transfer term $\bar{\alpha}$ was assumed to be a linear function of the micropore hydraulic conductivity, with two values considered to investigate its impact on the hydromechanical response: (a) $\bar{\alpha} / k_{m}=10^{2} \mathrm{~s} / \mathrm{m}$, and (b) $\bar{\alpha} / k_{m}=10^{3} \mathrm{~s} / \mathrm{m}$.

The initial condition was established, first, by subjecting the material to an isotropic compressive stress of $5 \mathrm{kPa}$, after which it was loaded to a vertical compressive stress of $200 \mathrm{kPa}$ under fully drained condition on both the macropores and micropores. Then, an additional downward load of $w=10 \mathrm{kPa}$ was applied on top of the mesh while allowing fluid in the macropores to drain to the top surface and fluid in the micropores to drain into the macropores. In other words, the top surface was assumed to be a zero-pressure boundary for the macropores and a no-flow boundary for the micropores, which means that the micropores could only drain into the macropores but not through the top drainage boundary. The consolidation phase began with a time increment of $1 \mathrm{~s}$ and with subsequent increments magnified by a factor of 1.01, i.e., $\Delta t_{n+1}=1.01 \Delta t_{n}$.

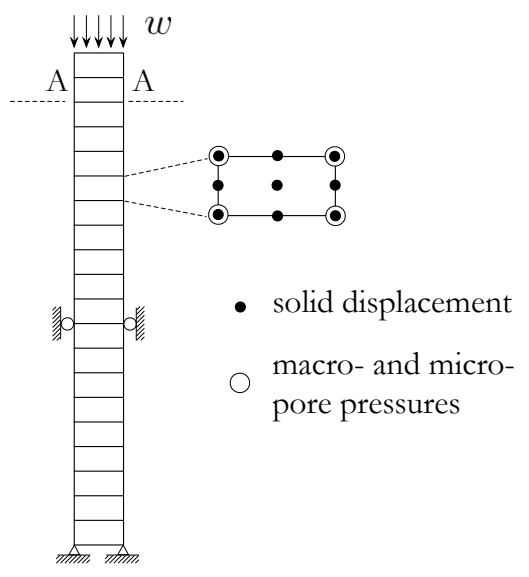

Fig. 10. Finite element mesh for $1 \mathrm{D}$ consolidation with secondary compression.

Figure 11 shows the time evolution of the vertical displacement at a point located $0.1 \mathrm{~m}$ from the top boundary (level A-A in Fig. 10). The shape of the curves suggest two stages of consolidation: a primary consolidation in which the macropores drain through the top boundary, followed by a secondary compression in which the micropores drain into the macropores. Note that for a sufficiently large mass transfer coefficient $\left(\bar{\alpha} / k_{m}=10^{3} \mathrm{~s} / \mathrm{m}\right)$ the secondary compression is not so pronounced; however, for a sufficiently small mass transfer coefficient $\left(\bar{\alpha} / k_{m}=10^{2} \mathrm{~s} / \mathrm{m}\right)$ the two stages of consolidation become very 
distinct. In the latter case, the first stage of consolidation had enough time to finish even before the second stage of compression could begin, producing a clear separation of scales in time domain.

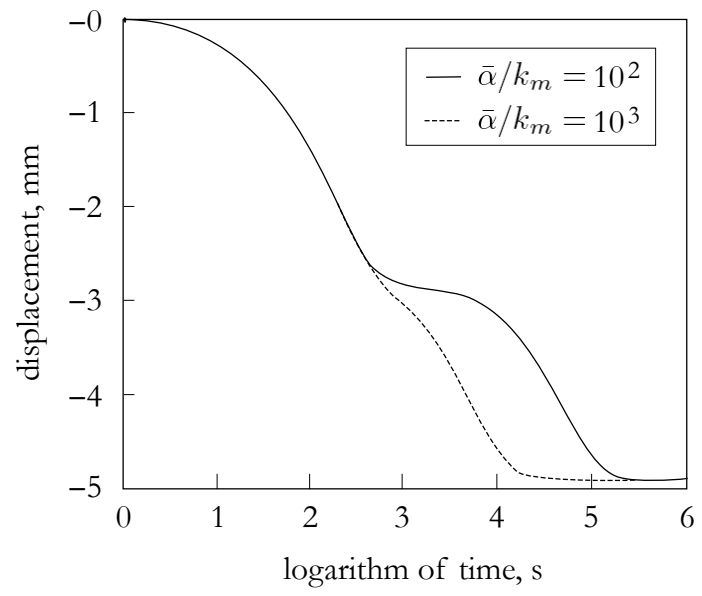

Fig. 11. Time evolution of vertical displacement at $0.1 \mathrm{~m}$ below the top boundary (level A-A) showing separation of scales. Values of $\bar{\alpha} / k_{m}$ in $\mathrm{s} / \mathrm{m}$.

For the same point at level A-A, Fig. 12 depicts the time evolution of the preconsolidation stresses $\bar{p}_{c}, p_{c M}$, and $p_{c m}$, relative to the initial value of $\bar{p}_{c}$. The macropore preconsolidation stress $p_{c M}$ shows early hardening as the transient macropore water pressure $p_{M}$ dissipates during the first stage of consolidation. However, the hardening response on $p_{c M}$ is followed by mild softening as the transient micropore water pressure $p_{m}$ dissipates and the micropore preconsolidation stress $p_{c m}$ undergoes hardening during the second stage of consolidation. Between $t=10^{5} \mathrm{~s}$ and $t=10^{6} \mathrm{~s}$, all three preconsolidation stresses approach a common value as the transient pore water pressures in both the macropores and micropores dissipate. Results of this example indeed show that the proposed double-porosity formulation can capture the secondary compression attributed to the fluid dissipation in the micropores.

Figure 13 portrays the spatial and temporal evolution of the internal structure variable $\psi$ for $\bar{\alpha} / k_{m}=10^{2} \mathrm{~s} / \mathrm{m}$. Between $t=10^{2} \mathrm{~s}$ and $t=10^{3} \mathrm{~s}$, the spatial distribution resembles what one would expect from a typical onedimensional consolidation curve, i.e., as the macropores compact, the micropore fraction $\psi$ increases. At $t=10^{3} \mathrm{~s}$, the primary consolidation is essentially complete, and $\psi$ is nearly uniform with depth. Beyond $t=10^{3} \mathrm{~s}$, secondary compression begins, producing nearly uniform compaction of the micropores with depth and a decrease in the value of $\psi$. Secondary compression appears to end at approximately $t=10^{6} \mathrm{~s}$. Once again, separation of scales is noted, 


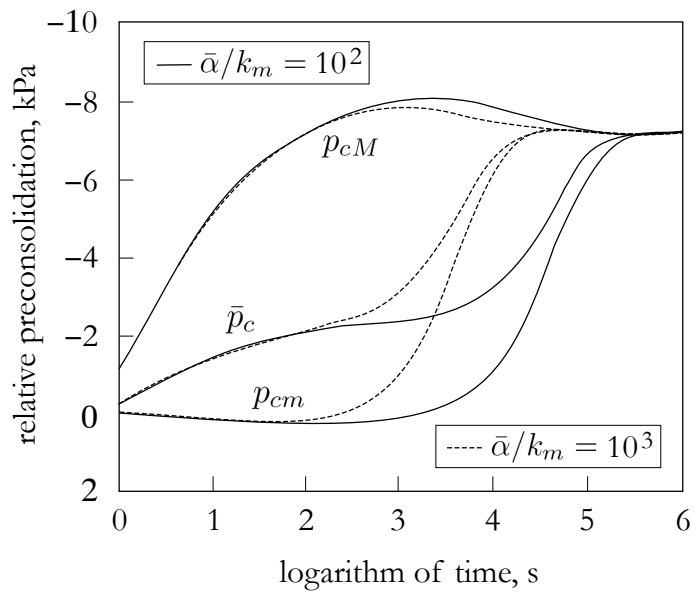

Fig. 12. Time evolution of preconsolidation stress, relative to the value of $\bar{p}_{c}$ at time $t=0$, at $0.1 \mathrm{~m}$ below the top boundary (level A-A). Values of $\bar{\alpha} / k_{m}$ in $\mathrm{s} / \mathrm{m}$.

in which the primary consolidation and secondary compression take place sequentially, due to the relatively small value of the mass transfer coefficient $\bar{\alpha}$ used in the simulation.

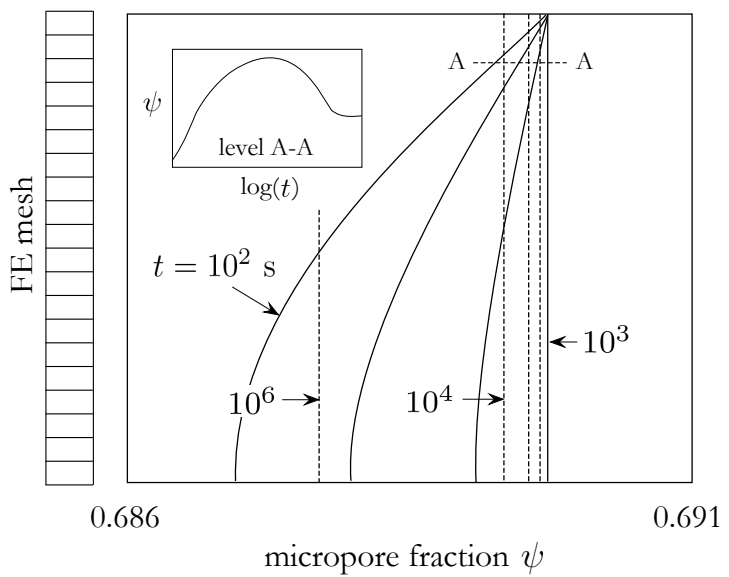

Fig. 13. Spatial and temporal evolution of $\psi$ alongside the finite element mesh. Level A-A is $0.1 \mathrm{~m}$ below the top. Solid curves denote primary consolidation; dashed lines denote secondary compression. 


\subsection{Secondary compression beneath a leaning tower}

The third and final example illustrates the effect of secondary compression on the time-dependent tilting of a seven-storey tower. The finite element mesh for this example accommodates a condition of plane strain and is shown in Fig. 14. The foundation of the tower consists of a $10 \mathrm{~m}$-thick sand layer underlain by a $30 \mathrm{~m}$-thick clay layer. Under the clay layer is a rigid, impermeable bedrock. The ground water table is located at the top of the sand layer. The tower is assumed to be rigid with a distributed weight of $W=6.6 \mathrm{MN} / \mathrm{m}$ at $23.2 \mathrm{~m}$ from the base. We should note that this example is only a hypothetical problem, and that the finite element mesh shown in Fig. 14 should not be interpreted as a model for any actual tower.

The sand layer is assumed to be a Neo-Hookean material with a bulk modulus of $12 \mathrm{MPa}$, Poisson's ratio of 0.3 , an effective (buoyant) unit weight of $1.0 \mathrm{Mg} / \mathrm{m}^{3}$, and a very high permeability. Hence, this layer may be assumed to be incapable of generating excess pore water pressure. The clay layer, on the other hand, is assumed to be a double-porosity material with similar properties to Pancone clay in Pisa, Italy, see [21]. Figure 15 shows the natural compressibility of this clay, as well as the compressibility of the reconstituted material. The fact that the two compressibility curves are not one on top of the other implies that this particular clay exhibits double porosity to a certain extent. Based on these compressibility curves, we have calibrated the material parameters for the clay as $c_{c}=0.176, c_{r}=0.022, c_{M}=0.6, \phi^{M}=$ 0.12 , and $\phi^{m}=0.52$. The intrinsic mass densities for the solid and water are assumed to be $2.65 \mathrm{Mg} / \mathrm{m}^{3}$ and $1.0 \mathrm{Mg} / \mathrm{m}^{3}$, respectively. The initial hydraulic conductivities of the macropores and micropores are assigned as $k_{M 0}=10^{-8}$ $\mathrm{m} / \mathrm{s}$ and $k_{m 0}=10^{-11} \mathrm{~m} / \mathrm{s}$, respectively, whereas the mass transfer coefficient has the value $\bar{\alpha} / k_{m}=100 \mathrm{~s} / \mathrm{m}$.

To begin the simulation, gravity load was first imposed on the foundation soil under 'drained' condition, i.e., with no excess pore fluid pressure developing. Since the Cam-Clay model does not allow a stress-free state, a very small initial isotropic compressive stress of $10 \mathrm{kPa}$ (effective) was first specified at all Gauss integrations points. Once the soil was in static equilibrium under gravity, the overconsolidation ratio of the clay was set to 1.6 (i.e., the size of the yield surface at yield was multiplied by a factor of 1.6) to define a preloaded state. Then, the tower load $W$ was applied under 'undrained' condition (i.e., no fluid flow). The tower was given a small geometrical tilt in the beginning so that by the time all of its weight was applied its inclination was around $\theta \approx 4.9^{\circ}$ from the vertical, see Fig. 14 . Under this sustained tower weight, the fluid pressure in the soil was allowed to dissipate, causing the tower to tilt further with time.

Figure 16 shows the time-evolution of the angle $\theta$ after all of the tower weight has been applied. For comparison, we also present the result obtained with single-porosity formulation in which the clay was assumed to have a hydraulic conductivity equal to the volume-average of the hydraulic conduc- 


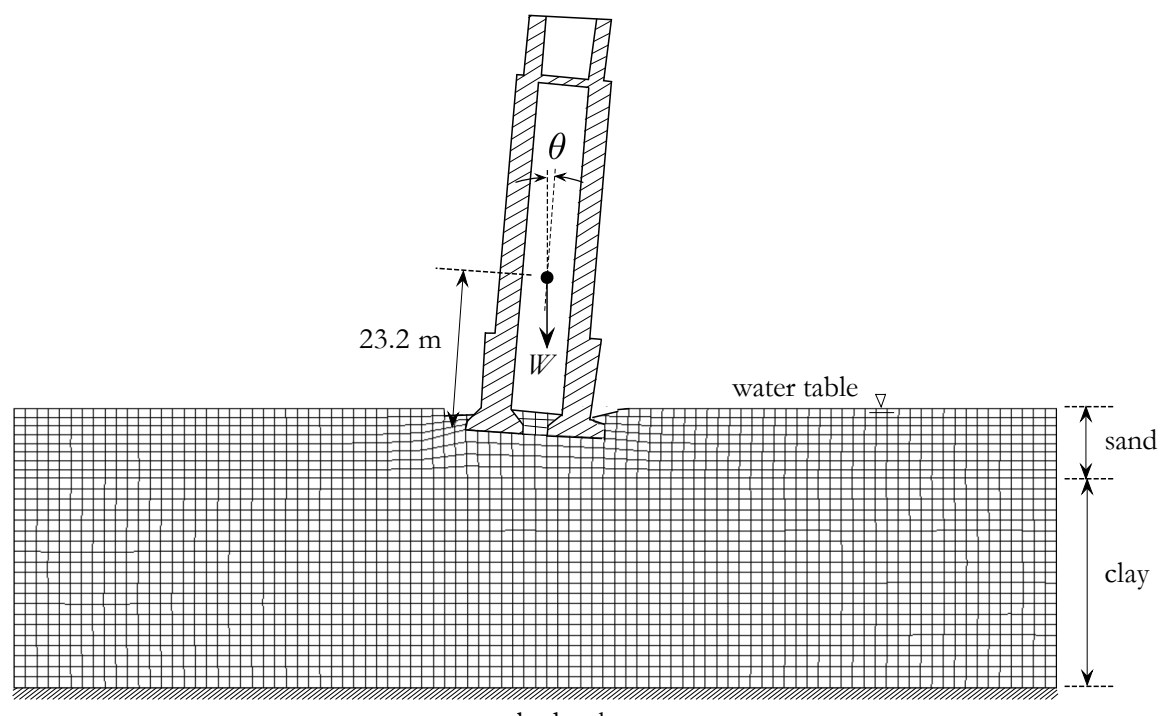

bedrock

Fig. 14. Finite element mesh for foundation of a leaning tower: clay layer is modeled as a double-porosity material with dual permeability.

tivities of the macropores and micropores. Observe that the double-porosity simulation predicts faster rate of fluid pressure dissipation, with the tower reaching a steady-state tilt at $t \approx 10^{3}$ days. This is to be expected since the higher permeability in the macropores would make drainage easier for the same drainage path, whereas the micropores would have a shorter effective drainage path since they could drain directly into the macropores. Also note that separation of scales in time is evident even on the inclination-time response, with the onset of secondary compression in the form of rapid change in slope noted at time $t \approx 20$ days. The double-porosity simulation also predicted slightly different tower tilt at steady state, resulting primarily from the fact that porosity changes in the macropores and micropores were tracked separately whereas the single-porosity simulation did not account for these changes explicitly.

Figures 17 and 18 show the time-evolution of the macropore and micropore fluid pressures, $p_{M}$ and $p_{m}$, respectively. Observe that $p_{M}$ appears to decrease monotonically with time everywhere (Fig. 17), whereas $p_{m}$ exhibits the so-called Mandel-Cryer effect in the vicinity of the tower base. This effect is characterized by a momentary increase in the fluid pressure (note the more intense red region at $t \approx 1$ day) before it subsequently dissipates (Fig. 18). On the other hand, Fig. 19 depicts the time-evolution of the internal structure 


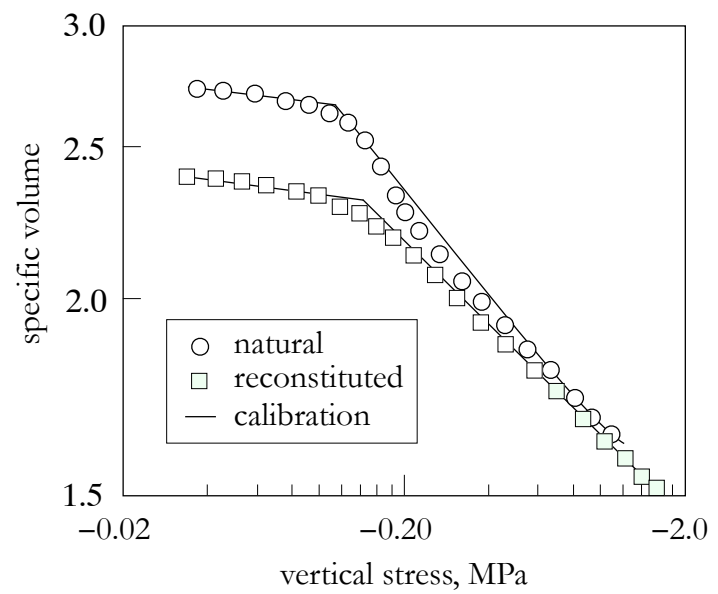

Fig. 15. Compressibility curves for natural and reconstituted Pancone clay. Experimental data from Callisto and Calabresi [21].

variable $\psi$. The figure suggests a significant increase of the micropore fraction in the vicinity of the tower base at time $t \approx 40$ days due to compression of the macropores, followed by a subsequent decrease at time $t \approx 400$ days due to compression of the micropores. In general, this sequential compression of the macropores and micropores is similar to the phenomenon of primary/secondary compression observed in the 1D consolidation example of the previous section.

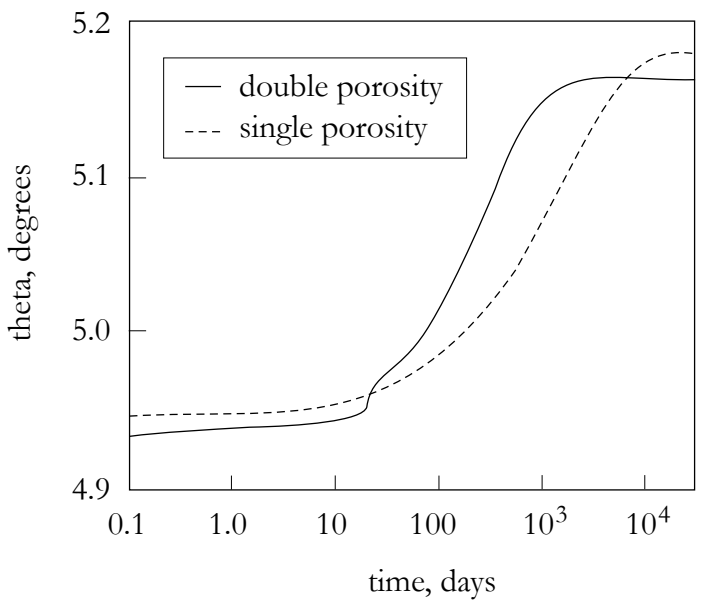

Fig. 16. Variation of tower inclination $\theta$ with time. 

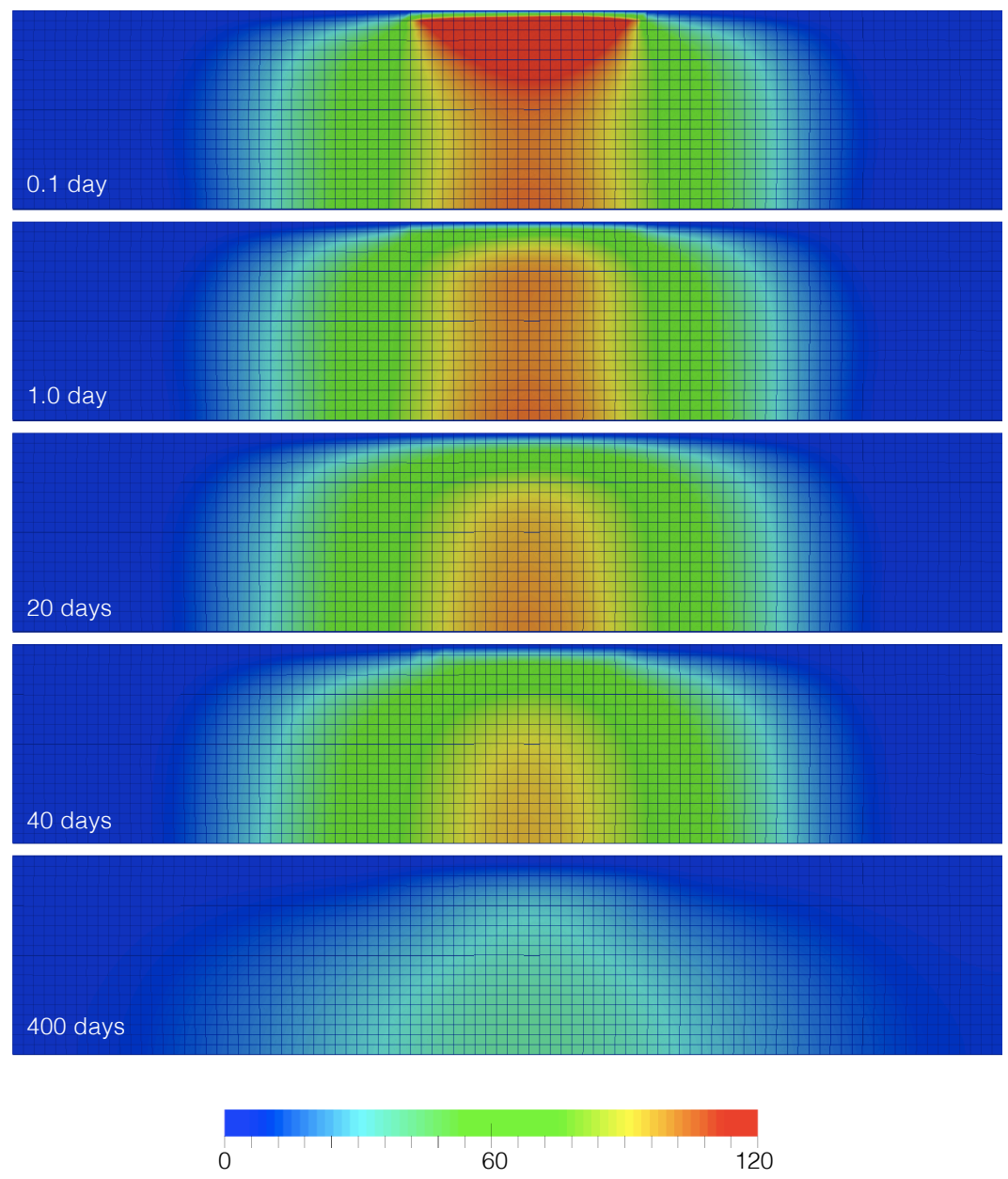

Fig. 17. Evolution of macropore pressure $p_{M}$ within the clay layer. Color bar in $\mathrm{kPa}$.

\section{Closure}

We have presented a novel constitutive framework for solid deformation and fluid flow in a porous material exhibiting two porosity scales. A unique feature of the framework is the separate treatment of hardening in the macropores and micropores arising from fluid flow through these two pore scales. This allows the evolution of the internal structure, herein represented by the micropore fraction $\psi$, to be defined concurrently with fluid flow and solid deformation. The formulation has been validated against experimental data on aggregated soils, and has been implemented into a finite element code 

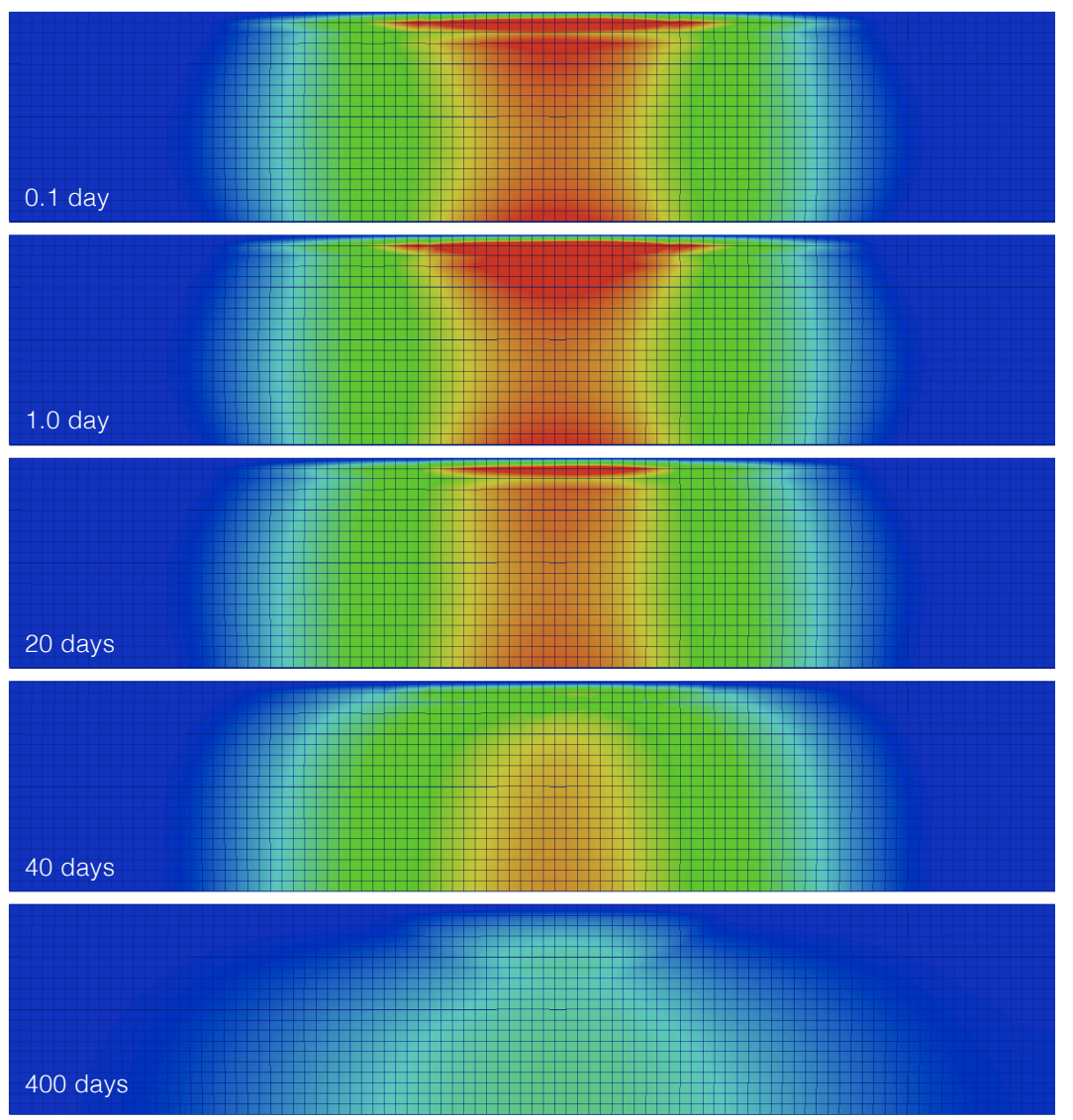

0

60

120

Fig. 18. Evolution of micropore pressure $p_{m}$ within the clay layer. Color bar in $\mathrm{kPa}$.

utilizing a $\boldsymbol{u} / p_{M} / p_{m}$ formulation. Many aggregated soils such as peat and highly compressible clays show pronounced secondary consolidation. This paper demonstrates that such phenomenon may be interpreted in the context of double-porosity problem as secondary compression attributed in part to delayed dissipation of excess pore pressures in the micropores, which is captured well by the proposed framework. 

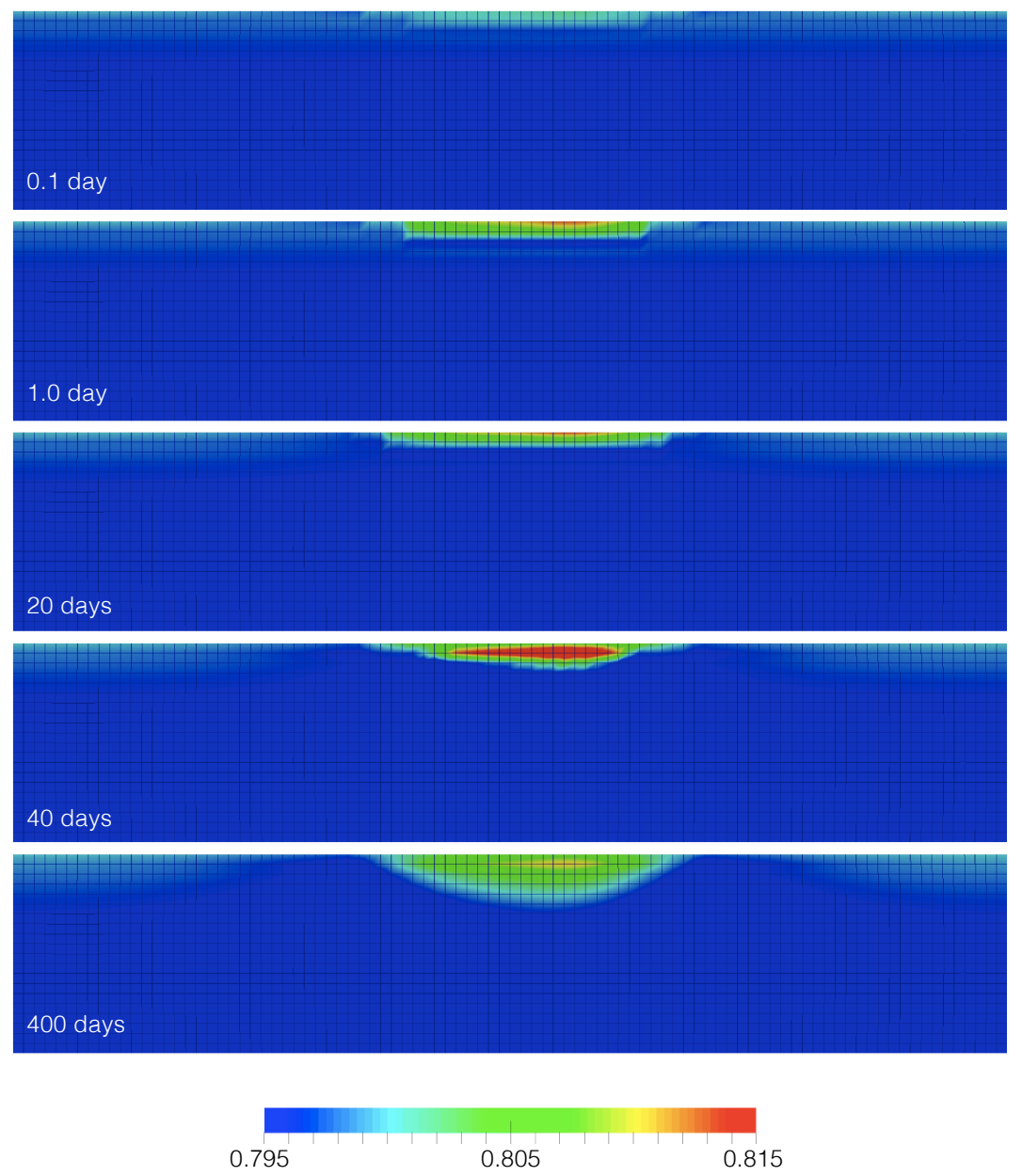

Fig. 19. Evolution of micropore fraction $\psi$ within the clay layer.

\section{Acknowledgements}

This material is based upon work supported by the U.S. Department of Energy, Office of Science, Office of Basic Energy Sciences, Geosciences Research Program, under Award Number DE-FG02-03ER15454; and by the National Science Foundation under Award Number CMMI-1462231. The second author acknowledges financial supports provided by the Fulbright Program, the John A. Blume Earthquake Engineering Center, and the Charles H. Leavell Graduate Student Fellowship. 


\section{Appendix A. Local tangent operator}

We first note the tangential elastic constitutive equation

$$
\delta \bar{\tau}_{A}=\sum_{B=1}^{3} a_{A B}^{\mathrm{e}} \delta \varepsilon_{B}^{\mathrm{e}},
$$

where $a_{A B}^{\mathrm{e}}=\partial^{2} \Psi^{\mathrm{e}} / \partial \varepsilon_{A}^{\mathrm{e}} \partial \varepsilon_{B}^{\mathrm{e}}$ is the tangential elasticity matrix in principal axes. Consider the local residual expression $\boldsymbol{r}=\boldsymbol{r}(\boldsymbol{x})$, where the components of $\boldsymbol{r}$ are given in (85)-(88) and those of $\boldsymbol{x}$ are given in (89). We note that for the local problem the values of $\varepsilon_{A}^{\mathrm{etr}}, p_{M}$, and $p_{m}$ are given.

The local tangent operator $\boldsymbol{A}=\boldsymbol{r}^{\prime}(\boldsymbol{x})$ has components given below. First, we consider the following derivatives with respect to $x_{A}=\varepsilon_{A}^{\mathrm{e}}$ for $A=1,2,3$ :

$$
A_{A B}=\delta_{A B}+\Delta \lambda \sum_{C=1}^{3} \frac{\partial^{2} F}{\partial \bar{\tau}_{A} \partial \bar{\tau}_{C}} a_{C B}^{\mathrm{e}}, \quad B=1,2,3 .
$$

The remaining derivatives for $A=1,2,3$ are

$$
A_{4 A}=\sum_{B=1}^{3} \frac{\partial F}{\partial \bar{\tau}_{B}} a_{B A}^{\mathrm{e}}, \quad A_{5 A}=-\psi \frac{\partial p_{c m}}{\partial \varepsilon_{A}^{\mathrm{e}}}, \quad A_{6 A}=\frac{\partial p_{c m}}{\partial \varepsilon_{A}^{\mathrm{e}}},
$$

where $\partial p_{c m} / \partial \varepsilon_{A}^{\mathrm{e}}=\partial p_{c m} / \partial \varepsilon_{v}^{\mathrm{e}}$.

Next we consider derivatives with respect to $x_{4}=\Delta \lambda$. For $A=1,2,3$, we have

$$
A_{A 4}=\frac{\partial F}{\partial \bar{\tau}_{A}}, \quad A_{44}=A_{54}=A_{64}=0 .
$$

The derivatives with respect to $x_{5}=\bar{p}_{c}$ are of the form (for $A=1,2,3$ )

$$
A_{A 5}=\Delta \lambda \frac{\partial^{2} F}{\partial \bar{\tau}_{A} \partial \bar{p}_{c}}, \quad A_{45}=\frac{\partial F}{\partial \bar{p}_{c}}, \quad A_{55}=1, \quad A_{65}=0 .
$$

Finally, the derivatives with respect to $x_{6}=\psi$ are of the form (for $A=$ $1,2,3,4)$

$$
\begin{aligned}
A_{A 6}=0, \quad A_{56} & =-\psi \frac{\partial p_{c m}}{\partial \psi}-p_{c m}-(1-\psi) \frac{\partial p_{c M}}{\partial \psi}+p_{c M}, \\
A_{66} & =\frac{\partial p_{c m}}{\partial \psi}-\frac{\partial p_{c M}}{\partial \psi} .
\end{aligned}
$$

We note from the chain rule that

$$
\frac{\partial p_{c M}}{\partial \psi}=\frac{\partial p_{c M}}{\partial e_{M}} \frac{\partial e_{M}}{\partial \psi}, \quad \frac{\partial p_{c m}}{\partial \psi}=\frac{\partial p_{c m}}{\partial v_{m}} \frac{\partial v_{m}}{\partial \psi} .
$$




\section{Appendix B. Global tangent operator}

This appendix develops the elements of the matrix $\boldsymbol{D}:=\partial \boldsymbol{r} / \partial \boldsymbol{z}$ necessary for constructing the global tangent operator. The components are $D_{A B}=$ $\partial r_{A} / \partial z_{B}$, where the ranges are $A \in[1,6]$ and $B \in[1,5]$. For $A, B=1,2,3$, we have

$$
\begin{aligned}
& D_{A B}=-\delta_{A B}, \quad D_{4 B}=0 \\
& D_{5 B}=-\psi \frac{\partial p_{c m}}{\partial \varepsilon_{B}^{\mathrm{etr}}}-(1-\psi) \frac{\partial p_{c M}}{\partial \varepsilon_{B}^{\mathrm{etr}}}, \\
& D_{6 B}=\frac{\partial p_{c m}}{\partial \varepsilon_{B}^{\mathrm{etr}}}-\frac{\partial p_{c M}}{\partial \varepsilon_{B}^{\mathrm{etr}}} .
\end{aligned}
$$

Continuing, we evaluate

$$
D_{A 4}=D_{A 5}=0, \quad \text { for } A=1, \ldots, 5, \quad D_{64}=1, \quad D_{65}=-1 .
$$

\section{References}

[1] Abousleiman, Y.N., Hoang, S.K. and Liu, C. (2014). Anisotropic porothermoelastic solution and hydro-thermal effects on fracture width in hydraulic fracturing. International Journal for Numerical and Analytical Methods in Geomechanics 38, 493-517.

[2] Al-Mukhtar, M. (1995). Macroscopic behavior and microstructural properties of a kaolinite clay under controlled mechanical and hydraulic state. In 1st International Conference on Unsaturated Soils / UNSAT 95, volume 1, pages 39, Paris.

[3] Alonso, E.E., Gens, A., and Josa, A. (1990). A constitutive model for partially saturated soils, Géotechnique 40, 405-430.

[4] Anagnostopoulos, A.G., Kalteziotis, N., Tsiambaos, G.K., and Kavvadas (1991). Geotechnical properties of the Corinth Canal marls. Geotechnical and Geological Engineering 9, 1-26.

[5] Argyris, J.H., Faust, G., Szimmat, J., Warnke, E.P., and Willam, K.J. (1974). Recent developments in the finite element analysis of prestressed concrete reactor vessels. Nuclear Engineering and Design 28, 42-75.

[6] Barrenblatt, G., Zheltov, I., and Kochina, I. (1960). Basic concepts in the theory of seepage of homogeneous liquids in fissured rocks. Journal of Applied Mathematics and Mechanics 24, 1286-1303.

[7] Barrenblatt, G. (1963). On certain boundary value problems for the equation of seepage of liquid in fissured rock. Journal of Applied Mathematics and Mechanics 27, 513-518.

[8] Bear, J. (1972). Dynamics of Fluids in Porous Media. Dover Publications, Inc., New York. 
[9] Behnia, M., Goshtasbi, K., Marji, M. F. and Golshani, A. (2015). Numerical simulation of interaction between hydraulic and natural fractures in discontinuous media. Acta Geotechnica 10, 533-546.

[10] Benkemoun, N., Gelet, R., Roubin, E. and Colliat, J.-B. (2015). Poroelastic two-phase material modeling: theoretical formulation and embedded finite element method implementation. International Journal for Numerical and Analytical Methods in Geomechanics 39, 1255-1275.

[11] Bennett, K.C., Berla, L.A., Nix, W.D. and Borja, R.I. (2015). Instrumented nanoindentation and 3D mechanistic modeling of a shale at multiple scales. Acta Geotechnica, 10, 1-14.

[12] Borja, R.I. (2013). Plasticity Modeling and Computation, SpringerVerlag, Berlin, Heidelberg.

[13] Borja, R.I., and Koliji, A. (2009). On the effective stress in unsaturated porous continua with double porosity. J. Mech. Phys. Solids 57, 11821193.

[14] Borja, R.I. (2006). On the mechanical energy and effective stress in saturated and unsaturated porous continua. Int. J. Solids Struct. 43, 17641786.

[15] Borja, R.I. (2004). Cam-Clay plasticity, Part V: A mathematical framework for three-phase deformation and strain localization analyses of partially saturated porous media. Comput. Methods Appl. Mech. Engrg. 193, 5301-5338.

[16] Borja, R.I., Tamagnini, C. and Alarcón, E. (1998). Elastoplastic consolidation at finite strain part 2: finite element implementation and numerical examples. Computer Methods in Applied Mechanics and Engineering $1259,103-122$.

[17] Borja, R.I. and Alarcón, E. (1995). A mathematical framework for finite strain elastoplastic consolidation Part 1: Balance laws, variational formulation, and linearization. Computer Methods in Applied Mechanics and Engineering 122, 145-171.

[18] Burland, J.B. (1990). On the compressibility and shear strength of natural clays, Géotechnique 40, 329-378.

[19] Butterfield, R. (1979). A natural compression law for soils, Géotehcnique $29,469-480$.

[20] Callari, C. and Federico, F. (2000). FEM validation of a double porosity elastic model for consolidation of structurally complex clayey soils. International Journal for Numerical and Analytical Methods in Geomechanics $24,367-402$.

[21] Callisto, L. and Calabresi, G. (1998). Mechanical behaviour of a natural soft clay, Géotechnique 48, 495-513.

[22] Carminati, A., Kaestner, A., Lehmann, P., and Flühler, H. (2008). Unsaturated water flow across soil aggregate contacts, Advances in Water Resources 31, 1221-1232. 
[23] Choo, J., and Borja, R.I. (2015). Stabilized mixed finite elements for deformable porous media with double porosity. Computer Methods in Applied Mechanics and Engineering 293, 131-154.

[24] Choo, J., White, J.A., and Borja, R.I. (2015). Hydromechanical modeling of unsaturated flow in double porosity media. International Journal of Geomechanics, in press.

[25] Cosenza, P., Korošak, D. (2014). Secondary consolidation of clay as an anomalous diffusion process. International Journal for Numerical and Analytical Methods in Geomechanics 38, 1231-1246.

[26] Delage, P., Audiguier, M., Cui, Y.-J., and Howat, M. D. (1996). Microstructure of compacted silt. Canadian Geotechnical Journal 33, 150158.

[27] Della Vecchia, G., Dieudonné, A-C., Jommi, C. and Charlier, R. (2014). Accounting for evolving pore size distribution in water retention models for compacted clays. International Journal for Numerical and Analytical Methods in Geomechanics 39, 702-723.

[28] Didwania, A.K. (2002). Micromemechanical basis of concept of effective stress. Journal of Engineering Mechanics 128, 864-868.

[29] Do, N.-A., Dias, D., Oreste, P., and Djeran-Maigre. I. (2014). Threedimensional numerical simulation for mechanized tunnelling in soft ground: the influence of the joint pattern. Acta Geotechnica 9, 673-694.

[30] Dykhuizen, R.C. (1990). A new coupling term for dual-porosity models. Water Resources Research 26, 351-356.

[31] Gens, A., Guimarães, L.N., Sánchez, M., and Valleján, B. (2011). Coupled analysis of double porosity swelling clays. In: R.I. Borja (Ed.) Multiscale and Multiphysics Processes in Geomechanics, Springer-Verlag, pp. 85-88.

[32] Gerke, H. H. (2006). Preferential flow descriptions for structured soils. Journal of Plant Nutrition and Soil Science 169, 382-400.

[33] Gerke, H.H., van Genuchten, M.T. (1993). A dual-porosity model for simulating the preferential movement of water and solutes in structured porous media. Water Resources Research 29, 305-319,

[34] Goudarzi, M. and Mohammadi, S. (2014). Weak discontinuity in porous media: an enriched EFG method for fully coupled layered porous media. International Journal for Numerical and Analytical Methods in Geomechanics 38, 1792-1822.

[35] Gray, W. G. and Schrefler, B. A. (2007). Analysis of the solid phase stress tensor in multiphase porous media. International Journal for Numerical and Analytical Methods in Geomechanics 31, 541-581.

[36] Gray, W. G. and Schrefler, B. A. (2001). Thermodynamic approach to effective stress in partially saturated porous media. European Journal of Mechanics A/Solids 20, 521-538.

[37] Gudehus, G. (1973). Elastoplastische Stoffgleichungen für trockenen Sand. Ingenieur-Archiv. 42, 151-169. 
[38] Guo, N. and Zhao, J. (2016). Parallel hierarchical multiscale modelling of hydro-mechanical problems for saturated granular soil. Computer Methods in Applied Mechanics and Engineering 305, 37-61.

[39] Haggerty, R., Gorelick, S.M. (1995). Multiple-rate mass transfer for modeling diffusion and surface reactions in media with pore-scale heterogeneity. Water Resources Research 31, 2383-2400.

[40] Hassanizadeh, S. M. and Gray, W. G. (1990). Mechanics and thermodynamics of multiphase flow in porous-media including interphase boundaries. Advances in Water Resources 13, 169-186.

[41] Houlsby, G.T. (1997). The work input to an unsaturated granular material. Géotechnique 47, 193-196.

[42] Houlsby, G.T. (1979). The work input to a granular material. Géotechnique 29, 354-358.

[43] Hutter, K., Laloui, L., and Vulliet, L. (1999). Thermodynamically based mixture models of saturated and unsaturated soils. Mechanics of Cohesive-frictional Materials 4, 295-338.

[44] Kavvadas, M., and Amorosi, A. (2000). A constitutive model for structured soils. Géotechnique 50, 263-273.

[45] Khalili, N., Witt, R., Laloui, L., Vulliet, L., and Koliji, A. (2005). Effective stress in double porous media with two immiscible fluids. Geophys. Res. Lett. 32(L15309), doi:10.1029/2005GL023766.

[46] Khalili, N. and Valliappan, S. (1996). Unified theory of flow and deformation in double porous media. European Journal of Mechanics A/Solids $15,321-336$.

[47] Köhne, J.M., Mohanty, B.P., Šimůnek, J., Gerke, H.H. (2004). Numerical evaluation of a second-order water transfer term for variably saturated dual-permeability models. Water Resources Research 40, W07409.

[48] Koliji, A., Laloui, L., and Vulliet, L. (2010). Constitutive modeling of unsaturated aggregated soils. International Journal for Numerical and Analytical Methods in Geomechanics 34, 1846-1876.

[49] Koliji, A., Vulliet, L., and Laloui, L. (2010). Structural characterization of unsaturated aggregated soil. Canadian Geotechnical Journal 47, 297311.

[50] Koliji, A., Lehmann P., Vulliet L., Laloui L., Carminati A., Vontobel P., Hassanein R. (2009). Assessment of structural evolution of aggregated soil using neutron tomography, Water Resources Research 44, W00C07, doi:10.1029/2007WR006297.

[51] Koliji, A. (2008). Mechanical behaviour of unsaturated aggregated soils. PhD thesis, No. 4011, Ecole Polytechnique Fédérale de Lausanne.

[52] Koliji, A., Vulliet, L., and Laloui, L. 2008. New basis for the constitutive modelling of aggregated soils. Acta Geotech. 3, 61-69.

[53] Koliji, A., Laloui, L., Cusinier, O., and Vulliet, L. (2006). Suction induced effects on the fabric of a structured soil. Transport in Porous Media 64, $261-278$. 
[54] Liu, M.D., and Carter, J.P. (2002). A structured Cam Clay model. Canadian Geotechnical Journal 39, 1313-1332.

[55] Mehrabian A. and Abousleiman Y.N. (2015). Gassmann equations the constitutive relations for multiple-porosity and multiple-permeability poroelasticity with applications to oil and gas shale. International Journal for Numerical Analytical Methods in Geomechanics 39, 1547-1569.

[56] Meschke, G. and Leonhart, D. (2015). A Generalized Finite Element Method for hydro-mechanically coupled analysis of hydraulic fracturing problems using space-time variant enrichment functions. Computer Methods in Applied Mechanics and Engineering 290, 438-465.

[57] Murad, M., Guerreiro, J.N., Loula, A.F.D. (2001). Micromechanical computational modeling of secondary consolidation and hereditary creep in soils. Computer Methods in Applied Mechanics and Engineering 190, 1985-2016.

[58] Navarro, V., Alonso, E.E. (2001). Secondary compression of clays as a local dehydration process. Géotechnique 51, 859-869.

[59] Pao, W.K.S. and Lewis, R.W. (2002). Three-dimensional finite element simulation of three phase flow in a deforming fissured reservoir. Computer Methods in Applied Mechanics and Engineering 191, 2631-2659.

[60] Park, D.S. and Kutter, B. (2016). Sensitive bounding surface constitutive model for structured clay. International Journal for Numerical and Analytical Methods in Geomechanics, DOI: 10.1002/nag.2507.

[61] Pouya, A. (2015). A finite element method for modeling coupled flow and deformation in porous fractured media. International Journal for Numerical and Analytical Methods in Geomechanics, 39, 1836-1852.

[62] Remij, E.W., Remmers, J.J.C., Huyghe, J.M. and Smeulders, D.M.J. (2015). The enhanced local pressure model for the accurate analysis of fluid pressure driven fracture in porous materials. Computer Methods in Applied Mechanics and Engineering 286, 293-312.

[63] Sarma, P., Aziz, K. (2006). New transfer functions for simulation of naturally fractured reservoirs with dual porosity models. SPE Journal 11, $328-340$.

[64] Semnani, S.J., White, J.A., Borja, R.I. (2016). Thermoplasticity and strain localization in transversely isotropic materials based on anisotropic critical state plasticity. International Journal for Numerical and Analytical Methods in Geomechanics, DOI: 10.1002/nag.2536.

[65] Shen, W., He, Z., Dormieux, L. and Kondo, D. (2014). Effective strength of saturated double porous media with a Drucker-Prager solid phase. International Journal for Numerical and Analytical Methods in Geomechanics 38, 281-296.

[66] Shi, X. S. and Herle, I. (2016) Analysis of the compression behavior of artificial lumpy composite materials. International Journal for Numerical and Analytical Methods in Geomechanics, DOI: 10.1002/nag.2493.

[67] Shi, X.S. and Herle, I. (2016). Numerical simulation of lumpy soils using a hypoplastic model. Acta Geotechnica, DOI: 10.1007/s11440-016-0447-7. 
[68] Silvestre, J.R., Vargas, Jr. E., Vaz, L.E. and Soares, A.C. (2015). Modelling of coupled fluid-mechanical problems in fractured geological media using enriched finite elements. International Journal for Numerical and Analytical Methods in Geomechanics 39, 1104-1140.

[69] Šimůnek, J., Jarvis, N. J., van Genuchten, M. T., and Gardenas, A. (2003). Review and comparison of models for describing non-equilibrium and preferential flow and transport in the vadose zone. Journal of Hydrology 272, 14-35.

[70] Song, X., Borja, R.I. (2014). Mathematical framework for unsaturated flow in the finite deformation range. International Journal for Numerical Methods in Engineering 14, 658-682.

[71] Song, X., Borja, R.I. (2014). Finite deformation and fluid flow in unsaturated soils with random heterogeneity. Vadose Zone Journal 13, DOI: 10.2136/vzj2013.07.0131,

[72] Sun, W., Chen, Q. and Ostien, J.T. (2014). Modeling the hydromechanical responses of strip and circular punch loadings on watersaturated collapsible geomaterials. Acta Geotechnica 9, 903-934

[73] Sun, W. (2015). A stabilized finite element formulation for monolithic thermo-hydro-mechanical simulations at finite strain, International Journal for Numerical Methods in Engineering 103, 798-839.

[74] Tjioe, M., and Borja, R.I. (2015). On the pore-scale mechanisms leading to brittle and ductile deformation behavior of crystalline rocks. International Journal for Numerical and Analytical Methods in Geomechanics $39,1165-1187$.

[75] Tjioe, M., and Borja, R.I. (2016). Pore-scale modeling of deformation and shear band bifurcation in porous crystalline rocks. International Journal for Numerical Methods in Engineering, DOI: 10.1002/nme.5208.

[76] Trottier, N., Delay, F., Bildstein, O. and Ackerer, P. (2014). Inversion of a dual-continuum approach to flow in a karstified limestone: Insight into aquifer heterogeneity revealed by well-test interferences. Journal of Hydrology 508, 157-169.

[77] Vu, M.-N., Pouya, A. and Seyedi, D.M. (2014). Theoretical and numerical study of the steady-state flow through finite fractured porous media. International Journal for Numerical and Analytical Methods in Geomechanics 38, 221-235.

[78] Wang, K. and Sun, W. (2016). A semi-implicit discrete-continuum coupling method for porous media based on the effective stress principle at finite strain. Computer Methods in Applied Mechanics and Engineering 304, 546-583.

[79] Wang, L., Pouya, A., Bornert, M. and Halphen, B. (2014). Modelling the internal stress field in argillaceous rocks under humidification/desiccation. International Journal for Numerical and Analytical Methods in Geomechanics 38, 1664-1682.

[80] Warren, J., Root, P. (1963). The behavior of naturally fractured reservoirs. SPE Journal 3, 245-255. 
[81] White, J.A., Castelletto, N. and Tchelepi, H.A. (2016). Block-partitioned solvers for coupled poromechanics: A unified framework. Computer Methods in Applied Mechanics and Engineering 303, 55-74.

[82] Xie, K.-H., Xia, C.-Q., An, R., Ying, H.-W. and Wu, H. (2016). A study on one-dimensional consolidation of layered structured soils. International Journal for Numerical and Analytical Methods in Geomechanics 40, 1081-1098.

[83] Zhu, C. and Arson, C. (2014). A thermo-mechanical damage model for rock stiffness during anisotropic crack opening and closure. Acta Geotechnica $9,847-867$. 